\title{
PENALIZED MODEL-BASED CLUSTERING OF FMRI DATA
}

\author{
A PREPRINT
}

\author{
Andrew DiLernia*1, Karina Quevedo ${ }^{2}$ Jazmin Camchong $^{2}$, \\ Kelvin Lim ${ }^{2}$, Wei Pan ${ }^{1}$, Lin Zhang ${ }^{1}$ \\ ${ }^{1}$ Division of Biostatistics, University of Minnesota, Minneapolis, MN, U.S.A. \\ ${ }^{2}$ Department of Psychiatry, University of Minnesota, Minneapolis, MN, U.S.A. \\ *diler001@umn.edu
}

October 14, 2020

\begin{abstract}
Functional magnetic resonance imaging (fMRI) data have become increasingly available and are useful for describing functional connectivity (FC), the relatedness of neuronal activity in regions of the brain. This $\mathrm{FC}$ of the brain provides insight into certain neurodegenerative diseases and psychiatric disorders, and thus is of clinical importance. To help inform physicians regarding patient diagnoses, unsupervised clustering of subjects based on FC is desired, allowing the data to inform us of groupings of patients based on shared features of connectivity. Since heterogeneity in FC is present even between patients within the same group, it is important to allow subject-level differences in connectivity, while still pooling information across patients within each group to describe group-level FC. To this end, we propose a random covariance clustering model (RCCM) to concurrently cluster subjects based on their FC networks, estimate the unique FC networks of each subject, and to infer shared network features. Although current methods exist for estimating FC or clustering subjects using fMRI data, our novel contribution is to cluster or group subjects based on similar FC of the brain while simultaneously providing group- and subject-level FC network estimates. The competitive performance of RCCM relative to other methods is demonstrated through simulations in various settings, achieving both improved clustering of subjects and estimation of FC networks. Utility of the proposed method is demonstrated with application to a resting-state fMRI data set collected on 43 healthy controls and 61 participants diagnosed with schizophrenia.
\end{abstract}

Keywords Brain connectivity - fMRI - Gaussian graphical models - Machine learning · Model-based clustering $\cdot$ Neuroimaging $\cdot$ Schizophrenia. 


\section{Introduction}

The availability of functional magnetic resonance imaging (fMRI) data has steadily increased, with demand for relevant analytical methods increasing accordingly. Useful for describing brain activity, the blood oxygen-level dependent (BOLD) signal underlying fMRI serves as a surrogate for neuronal activity [Menon et al., 1992]. These BOLD signals are often collected at thousands of three-dimensional cubes called voxels across time, yielding high-dimensional data. Dimension reduction prior to analysis is often done by averaging activation levels within sets of voxels called regions of interest (ROIs). Using these ROIs, one approach for analyzing fMRI data is to describe functional connectivity (FC), defined as the temporal dependence of neuronal activity in regions of the brain [Friston et al., 1993]. Alterations in FC have been associated with psychiatric disorders such as major depressive disorder [Zhu et al., 2012, Zeng et al., 2014] and schizophrenia [Yoon et al., 2008, Zhou et al., 2008, Pettersson-Yeo et al., 2011, Camchong et al., 2011, Fornito et al., 2012] as well as neurodegenerative diseases such as Alzheimer's [Dennis and Thompson, 2014], so describing altered FC among patients is of clinical importance.

For resting-state fMRI data from a single subject, graphical modeling approaches have commonly been used for joint analysis of FC connections among a set of ROIs [Lee et al., 2013, Smitha et al., 2017]. Gaussian graphical models (GGM) assume a multivariate Gaussian distribution for the BOLD signals, under which the inverse covariance matrix, called the precision matrix, conveys conditional dependencies between ROIs [Lauritzen, 2004], and is used to describe FC through a graphical representation of the ROI dependence structure. Specifically, an undirected graph is constructed with nodes representing ROIs, and edges connecting pairs of conditionally dependent nodes. In this setting, methods for estimating sparse precision matrices have been used to obtain the FC structure among ROIs for a single subject. One commonly used method is the graphical lasso or GLasso [Friedman et al., 2007]. For multi-subject fMRI data, there are often shared features of FC across subjects that can be accounted for to improve estimation. Extending the GLasso, many have proposed methods for jointly estimating multiple sparse precision matrices [Guo et al., 2011, Zhu et al., 2014, Danaher et al., 2014, Cai et al., 2016, Qiu et al., 2016, Fan et al., 2018, Zhang et al., 2020], which can be used for inference of multiple subject-level FC networks for multi-subject fMRI data. However, these methods do not account for between-subject heterogeneity in FC structures that is commonly present [Fiecas et al., 2017, Price et al., 2017]. This heterogeneity among subjects could be due to the presence of multiple subgroups or clusters of subjects with varying FC patterns [Mueller et al., 2013].

Although extensive work has been done on methods for classifying subjects based on resting-state and task-based fMRI signals [Davatzikos et al., 2005, Calhoun et al., 2008, Shen et al., 2010, Arribas et al., 2010, Castro et al., 2014], fewer studies have focused on unsupervised clustering of subjects based on FC patterns. Using an unsupervised maximum margin clustering method, Zeng et al. [2014] distinguished depressed patients from healthy controls based on FC estimates obtained from pairwise correlations. However, the maximum margin clustering approach does not yield interpretable estimates of FC network structures for each class or subject which are of clinical importance. Motivated by gene network analysis of microarray data, penalized Gaussian mixture models (GMM) have been proposed for simultaneous subject clustering and multiple network estimation [Zhou et al., 2009, Hill and Mukherjee, 2013, Gao et al., 2016, Hao et al., 2018]. However, these methods apply to subject clustering with only one observation per subject as often seen in gene expression data and assume a common covariance matrix for observations within a cluster. Thus, they are not applicable to multi-subject fMRI data which have multiple observations per subject, and they do not allow for within-cluster heterogeneity. 
In this paper, we propose a penalized model-based clustering method for resting-state fMRI data which groups patients based on FC features. Specifically, we propose a random covariance clustering method (RCCM) to simultaneously cluster subjects and obtain sparse precision matrix estimates for each cluster as well as each subject, producing interpretable estimates of subject- and group-level FC networks. A major contribution of the RCCM over existing penalized model-based clustering methods is to allow for clustering of entire subsets of fMRI data observed for each subject, rather than only individual observations, and that it allows subjects within each cluster to have similar but not identical FC networks. This is implemented via a hierarchical structure, in which the subject-level precision matrices follow a mixture of Wishart distributions, each with mean matrix equal to a corresponding cluster-level precision matrix. Using a mixture of Wishart distributions for each subject's precision matrix rather than a Gaussian mixture for individual observations retains observations together for each participant. This is necessary for clustering participants based on fMRI data since it would not be interpretable to have observations from a single participant separated into multiple clusters. The degrees of freedom of each Wishart component controls the level of similarity between each subject-level matrix and its corresponding group-level matrix and is treated as a tuning parameter which is selected via an extended stability approach to regularization selection (stARS) method described in Section 2.3. Our simulations provide evidence that by conducting concurrent clustering and network estimation our proposed RCCM has improved performance in the estimation of subject-level networks due to sharing information across similar subjects, while better clustering is achieved due to improved subject-level estimates. We applied the RCCM to a resting-state fMRI data set collected on 61 participants diagnosed with schizophrenia and 43 healthy controls, finding a slight tendency for participants diagnosed with schizophrenia to be clustered together.

For the rest of the paper, we present the proposed RCCM, the computational algorithm, and the selection of tuning parameters and the number of clusters in Section 2. Then, we describe simulations conducted to explore the relative performance of the RCCM to competitive two-step methods in Section 3 and illustrate the utility of the RCCM on a resting-state fMRI data set in Section 4. Lastly, in Section 5 we conclude with a discussion of our findings.

\section{Method}

\subsection{Random Covariance Clustering Model}

We consider the setting in which we have collected fMRI data on $K$ subjects for $p$ different ROIs with $n_{k}$ observations or time points on the $k^{t h}$ subject. We let $y_{k j t}$ denote the $t^{t h}$ observation or time point of the $j^{t h}$ ROI for the $k^{t h}$ subject for $k=1, \ldots, K, j=1, \ldots, p$, and $t=1, \ldots, n_{k}$.

We assume that $\mathbf{y}_{\mathbf{k t}}=\left(y_{k 1 t}, \ldots, y_{k p t}\right)^{T} \sim \mathcal{N}_{p}\left(\boldsymbol{\mu}_{\mathbf{k}}, \boldsymbol{\Sigma}_{\mathbf{k}}\right)$ are independent $p$-dimensional Gaussian random variables with mean vector $\boldsymbol{\mu}_{\mathrm{k}}$ and covariance matrix $\boldsymbol{\Sigma}_{\mathbf{k}}$. Moreover, we assume that the precision matrix of $\mathbf{y}_{\mathbf{k t}}, \Omega_{\mathbf{k}}=\Sigma_{\mathbf{k}}{ }^{-1}$, follows a mixture Wishart distribution with $G$ components:

$$
\boldsymbol{\Omega}_{\mathbf{k}} \sim p\left(\boldsymbol{\Omega}_{\mathbf{k}} ;\left\{\boldsymbol{\Omega}_{\mathbf{0 g}}, \pi_{g}\right\}_{g=1}^{G}\right)=\sum_{g=1}^{G} \pi_{g} p_{g}\left(\boldsymbol{\Omega}_{\mathbf{k}} ; \lambda_{2}, \boldsymbol{\Omega}_{\mathbf{0 g}}\right)
$$

where $p_{g}\left(\boldsymbol{\Omega}_{\mathbf{k}} ; \lambda_{2}, \boldsymbol{\Omega}_{\mathbf{0 g}}\right)$ is the probability density function (PDF) of the $g^{\text {th }}$ component corresponding to a Wishart random matrix with degrees of freedom $\lambda_{2}$ and mean $\Omega_{0 \mathrm{~g}}$. We note that $\Omega_{\mathrm{k}}$ describes the FC of the $k^{t h}$ subject, $\Omega_{0 \mathrm{~g}}$ describes the cluster-level FC for the $g^{\text {th }}$ group, and that $\pi_{g}$ can be 
interpreted as the proportion of subjects belonging to cluster $g$ where $\sum_{g=1}^{G} \pi_{g}=1$ for $g=1, \ldots, G$. An essential element for the novelty of our model, this mixture Wishart distribution of the subjectlevel $\Omega_{k}$ facilitates an interpretation of each subject's FC being similar to their corresponding cluster-level FC, but not necessarily identical. The degrees of freedom $\lambda_{2}$ is a tuning parameter controlling the degree of similarity between subject and cluster-level precision matrices, with higher values of $\lambda_{2}$ inducing more similarity between each subject-level matrix and its corresponding grouplevel matrix. The hierarchy of our proposed RCCM is illustrated in Figure 1, which illustrates the three-level structure of our model: the cluster level, within-cluster subject level, and within-subject observation level. Heterogeneity is assumed to be present in both the cluster- and subject-levels, while the observations for each subject are assumed to be homogeneous.

Assuming without loss of generality that our observed data is centered so that $\boldsymbol{\mu}_{\mathrm{k}}=\mathbf{0}$ for $k=1, \ldots, K$, the model likelihood for our observed data is

$$
L=\prod_{k=1}^{K} \prod_{t=1}^{n_{k}}\left(f_{k}\left(\mathbf{y}_{\mathbf{k t}} ; \boldsymbol{\Omega}_{\mathbf{k}}\right)\right) p\left(\boldsymbol{\Omega}_{\mathbf{k}} ;\left\{\pi_{g}, \boldsymbol{\Omega}_{\mathbf{0 g}}\right\}_{g=1}^{G}\right)
$$

where $f_{k}\left(\mathbf{y}_{\mathbf{k t}} ; \boldsymbol{\Omega}_{\mathbf{k}}\right)=\frac{\left|\boldsymbol{\Omega}_{\mathbf{k}}\right|^{1 / 2}}{(2 \pi)^{p / 2}} \exp \left(-\frac{1}{2} \mathbf{y}_{\mathbf{k t}}^{\mathbf{T}} \boldsymbol{\Omega}_{\mathbf{k}} \mathbf{y}_{\mathbf{k t}}\right)$ is the PDF of a mean $\mathbf{0}$ multivariate normal, $|\boldsymbol{\Omega}|$ denotes the determinant of a matrix $\Omega$, and

$p\left(\boldsymbol{\Omega}_{\mathbf{k}} ;\left\{\pi_{g}, \boldsymbol{\Omega}_{\mathbf{0 g}}\right\}_{g=1}^{G}\right)=\sum_{g=1}^{G} \pi_{g} p_{g}\left(\boldsymbol{\Omega}_{\mathbf{k}} ; \lambda_{2}, \boldsymbol{\Omega}_{\mathbf{0 g}}\right)=\sum_{g=1}^{G} \pi_{g}\left(\frac{\left|\boldsymbol{\Omega}_{\mathbf{k}}\right|^{\frac{\lambda_{2}-p-1}{2}} \exp \left(-\operatorname{tr}\left(\lambda_{2} \boldsymbol{\Omega}_{\mathbf{0 g}}{ }^{-1} \boldsymbol{\Omega}_{\mathbf{k}}\right) / 2\right)}{2^{\lambda_{2} p / 2}\left|\frac{1}{\lambda_{2}} \boldsymbol{\Omega}_{\mathbf{0 g}}\right|^{\lambda_{2} / 2} \Gamma_{p}\left(\lambda_{2} / 2\right)}\right)$

is a Wishart mixture distribution with $G$ components where $\Gamma_{p}(\cdot)$ denotes the multivariate Gamma function. Hence, the corresponding model log-likelihood is

$$
\ell=\log (L)=\sum_{k=1}^{K} \sum_{t=1}^{n_{k}} \log \left(f_{k}\left(\mathbf{y}_{\mathbf{k t}} ; \boldsymbol{\Omega}_{\mathbf{k}}\right)\right)+\sum_{k=1}^{K} \log p\left(\boldsymbol{\Omega}_{\mathbf{k}} ;\left\{\pi_{g}, \boldsymbol{\Omega}_{\mathbf{0 g}}\right\}_{g=1}^{G}\right)
$$

and thus

$$
-2 \ell=\sum_{k=1}^{K} \sum_{t=1}^{n_{k}}\left(\mathbf{y}_{\mathbf{k t}}^{\mathbf{T}} \mathbf{\Omega}_{\mathbf{k}} \mathbf{y}_{\mathbf{k t}}-\log \left|\mathbf{\Omega}_{\mathbf{k}}\right|\right)-2 \sum_{k=1}^{K} \log \left(\sum_{g=1}^{G} \pi_{g} p_{g}\left(\boldsymbol{\Omega}_{\mathbf{k}} ; \lambda_{2}, \boldsymbol{\Omega}_{\mathbf{0 g}}\right)\right) .
$$




\begin{tabular}{|c|c|c|}
\hline Level & Model & Interpretation \\
\hline Cluster & $\left\{\boldsymbol{\Omega}_{\mathbf{0 g}}\right\}_{g=1}^{G}$ & $\begin{array}{l}\text { Set of } G \text { cluster-level precision } \\
\text { matrices describing group-level FC }\end{array}$ \\
\hline Subject & $\left\{\boldsymbol{\Omega}_{\mathbf{k}} \stackrel{i i d}{\sim} \sum_{g=1}^{G} \pi_{g} p_{g}\left(\boldsymbol{\Omega}_{\mathbf{k}} ; \lambda_{2}, \boldsymbol{\Omega}_{\mathbf{0 g}}\right)\right\}_{k=1}^{K}$ & $\begin{array}{l}\text { Set of } K \text { subject-level precision } \\
\text { matrices describing unique } \\
\text { individual-level FC }\end{array}$ \\
\hline Observation & $\left\{\left\{\mathbf{y}_{\mathbf{k t}} \mid \boldsymbol{\Omega}_{\mathbf{k}} \stackrel{i n d}{\sim} \mathcal{N}_{p}\left(\mu_{k}, \boldsymbol{\Omega}_{\mathbf{k}}\right)\right\}_{t=1}^{n_{k}}\right\}_{k=1}^{K}$ & $\begin{array}{l}\text { Set of fMRI signals consisting } \\
\text { of } n_{k} \text { observations of } p \text { variables } \\
\text { for subject } k \text { where } p \text { is the } \\
\text { number of ROI }\end{array}$ \\
\hline
\end{tabular}

Figure 1: Hierarchy of Random Covariance Clustering Model (RCCM).

To induce sparsity in our precision matrix estimates, we include lasso penalties on the subjectand cluster-level precision matrices with different regularization parameters. Thus, letting $\Theta=$ $\left\{\left(\pi_{g}, \Omega_{k}, \Omega_{0 g}\right)\right\}$ for $g=1, \ldots, G$ and $k=1, \ldots, K$ denote the set of unknown parameters given the number of clusters or groups, $G$, we aim to estimate $\Theta$ by minimizing the following penalized objective function:

$$
\begin{aligned}
\sum_{k=1}^{K} \sum_{t=1}^{n_{k}}\left(\mathbf{y}_{\mathbf{k t}}^{\mathbf{T}} \mathbf{\Omega}_{\mathbf{k}} \mathbf{y}_{\mathbf{k t}}-\log \left|\boldsymbol{\Omega}_{\mathbf{k}}\right|\right) & -2 \sum_{k=1}^{K} \log \left(\sum_{g=1}^{G} \pi_{g} p_{g}\left(\boldsymbol{\Omega}_{\mathbf{k}} ; \lambda_{2}, \boldsymbol{\Omega}_{\mathbf{0 g}}\right)\right)+\lambda_{1} \sum_{k=1}^{K}\left\|\boldsymbol{\Omega}_{\mathbf{k}}\right\|_{1} \\
& +\lambda_{3} \sum_{g=1}^{G}\left\|\boldsymbol{\Omega}_{\mathbf{0 g}}\right\|_{1},
\end{aligned}
$$

where $\|\Omega\|_{1}=\sum_{i \neq j}\left|\omega_{i, j}\right|$ gives the sum of the absolute value of off-diagonal entries of the matrix $\Omega$, and $\lambda_{1}$ and $\lambda_{3}$ are non-negative tuning parameters for the lasso penalties.

\subsection{Computational Algorithm}

\subsubsection{E-Step}

We seek to minimize the objective function in Equation (1) using the EM algorithm [Dempster et al., 1977]. For the E-step, we first introduce latent variables which are indicators of cluster membership. Specifically, we let $z_{g k}=\mathbb{1}\left\{\Omega_{\mathbf{k}} \sim p_{g}\left(\Omega_{\mathbf{k}} ; \lambda_{2}, \Omega_{\mathbf{0 g}}\right)\right\}=\mathbb{1}\{$ subject $k$ is from cluster $g\}$, and define

$$
w_{g k}=\operatorname{Pr}\left(z_{g k}=1 \mid \Theta\right)=\mathbb{E}\left[z_{g k} \mid \Theta\right]=\frac{\pi_{g} p_{g}\left(\boldsymbol{\Omega}_{\mathbf{k}} ; \lambda_{2}, \boldsymbol{\Omega}_{\mathbf{0 g}}\right)}{\sum_{c=1}^{G} \pi_{c} p_{c}\left(\boldsymbol{\Omega}_{\mathbf{k}} ; \lambda_{2}, \boldsymbol{\Omega}_{\mathbf{0 c}}\right)},
$$


where $\Theta=\left\{\Omega_{\mathbf{k}}, \pi_{g}, \Omega_{0 \mathrm{~g}}\right\}$ for $g=1, \ldots, G$ and $k=1, \ldots, K$, and $\mathbb{1}\{\cdot\}$ is the indicator function. Thus, our complete objective function is

$$
\sum_{k=1}^{K} \sum_{t=1}^{n_{k}}\left(\mathbf{y}_{\mathbf{k} \mathbf{t}}^{\mathbf{T}} \mathbf{\Omega}_{\mathbf{k}} \mathbf{y}_{\mathbf{k t}}-\log \left|\boldsymbol{\Omega}_{\mathbf{k}}\right|\right)-2 \sum_{k=1}^{K} \sum_{g=1}^{G} z_{g k}\left(\log \left(\pi_{g}\right)+\log \left(p_{g}\left(\boldsymbol{\Omega}_{\mathbf{k}} ; \lambda_{2}, \boldsymbol{\Omega}_{\mathbf{0 g}}\right)\right)\right)+\lambda_{1} \sum_{k=1}^{K}\left\|\boldsymbol{\Omega}_{\mathbf{k}}\right\|_{1}+\lambda_{3} \sum_{g=1}^{G}\left\|\boldsymbol{\Omega}_{\mathbf{0 g}}\right\|_{1}
$$

since $\sum_{g=1}^{G} z_{g k}=1$ for $k=1, \ldots, K$ as each subject belongs to one and only one cluster. Therefore, since $\mathbb{E}_{z}\left[z_{g k} \mid \Theta^{(r)}\right]=w_{g k}^{(r)}$ it follows that the conditional expectation of our complete objective function is

$$
\begin{aligned}
Q\left(\Theta ; \Theta^{(r)}\right) & =\sum_{k=1}^{K} \sum_{t=1}^{n_{k}}\left(\mathbf{y}_{\mathbf{k t}}^{\mathbf{T}} \boldsymbol{\Omega}_{\mathbf{k}} \mathbf{y}_{\mathbf{k t}}-\log \left|\boldsymbol{\Omega}_{\mathbf{k}}\right|\right)-2 \sum_{k=1}^{K} \sum_{g=1}^{G} w_{g k}^{(r)}\left(\log \left(\pi_{g}\right)+\log \left(p_{g}\left(\boldsymbol{\Omega}_{\mathbf{k}} ; \lambda_{2}, \boldsymbol{\Omega}_{\mathbf{0 g}}\right)\right)\right)+ \\
& \lambda_{1} \sum_{k=1}^{K}\left\|\boldsymbol{\Omega}_{\mathbf{k}}\right\|_{1}+\lambda_{3} \sum_{g=1}^{G}\left\|\boldsymbol{\Omega}_{\mathbf{0 g}}\right\|_{1} .
\end{aligned}
$$

\subsubsection{M-Step}

We now seek to minimize the $Q\left(\Theta ; \Theta^{(r)}\right)$ function in Equation 2 by simultaneously estimating $G$ cluster-level matrices and $K$ subject-level precision matrices. We use an expectation/conditional maximization approach for optimization [Meng and Rubin, 1993]. Specifically, we iteratively update two blocks: the set of subject-level precision matrices given by $\Omega=\left\{\Omega_{\mathbf{k}}\right\}_{k=1}^{K}$ and the set of cluster-level precision matrices given by $\Omega_{0}=\left\{\Omega_{0 \mathrm{~g}}\right\}_{g=1}^{G}$. We note that this objective function is non-convex, and due to the lasso penalties, we are no longer guaranteed to decrease our objective function at each iteration which could slow the method's convergence [Green, 1990]. We proceed with the algorithm as follows:

1. Initialize $\Omega_{\mathbf{k}}{ }^{(0)}=\hat{\Omega}_{\mathbf{k}_{\mathbf{g l}}}$ for $k=1, \ldots, K$ where $\hat{\Omega}_{\mathbf{k}_{\mathrm{gl}}}$ is the individual GLasso precision matrix estimate for subject $k$ using a regularization parameter of 0.001 .

2. Initialize $w_{g k}^{(0)}$ to be 0 or 1 based on a hard assignment of $\left\{\Omega_{\mathbf{k}}{ }^{(0)}\right\}_{k=1}^{K}$ into $G$ disjoint clusters. Cluster assignments could be random, but we determined them using a Ward hierarchical clustering method implemented via the hclust function from the stats package in R, using a matrix of the Frobenius norm of pair-wise differences as a distance matrix [Murtagh and Legendre, 2014, R Core Team, 2018].

3. Update each $\pi_{g}$ by calculating $\pi_{g}^{(r+1)}=\frac{1}{K} \sum_{k=1}^{K} w_{g k}^{(r)}$ for $g=1, \ldots, G$.

4. Update each $\Omega_{0 \mathrm{~g}}$ by calculating

$$
\boldsymbol{\Omega}_{\mathbf{0 g}}{ }^{(r+1)}=\min _{\boldsymbol{\Omega}_{\mathbf{0 g}}}\left\{\operatorname{tr}\left(\frac{\sum_{k=1}^{K} w_{g k}^{(r)} \boldsymbol{\Omega}_{\mathbf{k}}^{(r)}}{\sum_{k=1}^{K} w_{g k}^{(r)}} \boldsymbol{\Omega}_{\mathbf{0 g}}{ }^{-1}\right)+\log \left|\boldsymbol{\Omega}_{\mathbf{0 g}}\right|+\frac{\lambda_{3}}{\lambda_{2} \sum_{k=1}^{K} w_{g k}^{(r)}}\left\|\boldsymbol{\Omega}_{\mathbf{0 g}}\right\|_{1}\right\}
$$


using Bien and Tibshirani's (2011) majorize-minimize algorithm for solving the covariance graphical lasso.

5. Update each $w_{g k}$ by calculating

$$
w_{g k}^{(r+1)}=\frac{\pi_{g}^{(r+1)} \exp \left(-\frac{\lambda_{2}}{2} \operatorname{tr}\left(\boldsymbol{\Omega}_{\mathbf{0 g}}^{(r+1)^{-1}} \boldsymbol{\Omega}_{\mathbf{k}}^{(r)}\right)\right)\left|\Omega_{\mathbf{0 g}}{ }^{(r+1)}\right|^{-\frac{\lambda_{2}}{2}}}{\sum_{c=1}^{G} \pi_{c}^{(r+1)} \exp \left(-\frac{\lambda_{2}}{2} \operatorname{tr}\left(\boldsymbol{\Omega}_{\mathbf{0 c}}^{(r+1)^{-1}} \boldsymbol{\Omega}_{\mathbf{k}}^{(r)}\right)\right)\left|\boldsymbol{\Omega}_{\mathbf{0 c}}{ }^{(r+1)}\right|^{-\frac{\lambda_{2}}{2}}},
$$

for $k=1, \ldots, K$ and $g=1, \ldots, G$.

6. Update each $\Omega_{\mathbf{k}}$ by calculating

$$
\boldsymbol{\Omega}_{\mathbf{k}}^{(r+1)}=\min _{\boldsymbol{\Omega}_{\mathbf{k}}}\left\{\operatorname{tr}\left(\frac{n_{k} \mathbf{S}_{\mathbf{k}}+\lambda_{2} \sum_{g=1}^{G} w_{g k}^{(r)} \boldsymbol{\Omega}_{\mathbf{0 g}}^{(r+1)^{-1}}}{n_{k}+\lambda_{2}-p-1} \boldsymbol{\Omega}_{\mathbf{k}}\right)-\log \left|\boldsymbol{\Omega}_{\mathbf{k}}\right|+\frac{\lambda_{1}}{n_{k}+\lambda_{2}-p-1}\left\|\boldsymbol{\Omega}_{\mathbf{k}}\right\|_{1}\right\}
$$

using the GLasso algorithm of Friedman et al. [2007], where $\mathbf{S}_{\mathbf{k}}=\frac{1}{n_{k}} \sum_{t=1}^{n_{k}} \mathbf{y}_{\mathbf{k t}} \mathbf{y}_{\mathbf{k t}}^{\mathbf{T}}$ for $k=1, \ldots, K$.

7. Update each $w_{g k}$ by calculating

$$
w_{g k}^{(r+1)}=\frac{\pi_{g}^{(r+1)} \exp \left(-\frac{\lambda_{2}}{2} \operatorname{tr}\left(\boldsymbol{\Omega}_{\mathbf{0 g}}^{(r+1)^{-1}} \boldsymbol{\Omega}_{\mathbf{k}}^{(r+1)}\right)\right)\left|\frac{1}{\lambda_{2}} \boldsymbol{\Omega}_{\mathbf{0 g}}^{(r+1)}\right|^{-\frac{\lambda_{2}}{2}}}{\sum_{c=1}^{G} \pi_{c}^{(r+1)} \exp \left(-\frac{\lambda_{2}}{2} \operatorname{tr}\left(\boldsymbol{\Omega}_{\mathbf{0 c}}^{(r+1)^{-1}} \boldsymbol{\Omega}_{\mathbf{k}}^{(r+1)}\right)\right)\left|\frac{1}{\lambda_{2}} \boldsymbol{\Omega}_{\mathbf{0 c}}^{(r+1)}\right|^{-\frac{\lambda_{2}}{2}}},
$$

for $k=1, \ldots, K$ and $g=1, \ldots, G$.

8. Repeat steps 3 through 7 until convergence as determined by

$$
\max \left\{\left\{\left|\boldsymbol{\Omega}_{k_{(i, j)}}^{(r+1)}-\boldsymbol{\Omega}_{k_{(i, j)}}^{(r)}\right|\right\} \cup\left\{\left|\boldsymbol{\Omega}_{g_{(i, j)}}^{(r+1)}-\boldsymbol{\Omega}_{g_{(i, j)}}^{(r)}\right|\right\}\right\}<\varepsilon,
$$

where $\varepsilon>0$ is a small number. That is, convergence is achieved when the largest change in magnitude of all individual entries of both the subject- and group-level estimates is smaller than $\varepsilon$, where we used $\varepsilon=0.001$. Derivations of updates are included in Appendix A.

\subsection{Selection of Tuning Parameters and Number of Clusters}

There are three tuning parameters for the proposed RCCM denoted $\lambda_{1}, \lambda_{2}$, and $\lambda_{3}$ which control the sparsity of subject-level precision matrices, the within-cluster variability, and the sparsity of clusterlevel precision matrices, respectively. Methods such as cross-validation (CV) and information criterion such as AIC and BIC can be used to determine the values of these tuning parameters, but we found for our real data analysis that $\mathrm{CV}$ yielded network estimates that were too dense with nearly all nodes connected to one another, while AIC yielded network estimates that were too sparse. 
Instead, we propose a modified stability approach for regularization selection (stARS) method [Liu et al., 2010]. The stARS method uses a sub-sampling approach to select the tuning parameter that yields the least amount of regularization while still obtaining an estimate that is sparse and stable across subsamples. Although Liu et al. [2010] implemented stARS for a single-subject graphical lasso problem, we extend the approach to our RCCM method in the context of analyzing data from multiple subjects. Specifically, given a sample of $n_{k}$ observations for $k=1, \ldots, K$, our extended stARS method consists of the following steps:

1. For $k=1, \ldots, K$, draw $N$ distinct number of subsamples without replacement from the $n_{k}$ total observations denoted $S_{k 1}, \ldots, S_{k N}$, each of size $b\left(n_{k}\right)=\left\lfloor 10 \sqrt{n_{k}}\right\rfloor$, where $\lfloor\cdot\rfloor$ denotes the floor function.

2. For each candidate tuning parameter value, implement the desired method to obtain subjectlevel precision matrix estimates for each subsample yielding $N$ estimated edge sets for each subject denoted by $\hat{E}_{k 1}(\Lambda), \ldots, \hat{E}_{k N}(\Lambda)$, where $\Lambda=\left(\lambda_{1}, \lambda_{2}, \lambda_{3}\right)$.

3. Calculate

$$
\hat{\theta}_{k ; s t}(\Lambda)=\frac{1}{N} \sum_{j=1}^{N} \mathbb{1}\left\{\hat{E}_{k j ; s t}\right\}
$$

where $\mathbb{1}\left\{\hat{E}_{k j ; s t}\right\}=1$ if the $j^{\text {th }}$ subsample implies an edge between nodes $s$ and $t$ for the $k^{t h}$ subject and 0 otherwise. Thus, $\hat{\theta}_{k ; s t}(\Lambda)$ is the proportion of subsamples with an edge between $s$ and $t$ for the $k^{\text {th }}$ subject.

4. Calculate $\hat{\xi}_{k ; s t}(\Lambda)=2 \hat{\theta}_{k ; s t}(\Lambda)\left(1-\hat{\theta}_{k ; s t}(\Lambda)\right)$, which can be interpreted as the proportion of times that each pair of subsamples disagrees on the presence of an edge between nodes $s$ and $t$ for the $k^{t h}$ subject.

5. Calculate $\hat{D}(\Lambda)=\frac{1}{K} \sum_{k=1}^{K} \sum_{s<t} \hat{\xi}_{k ; s t}(\Lambda) /\left(\begin{array}{l}p \\ 2\end{array}\right)$ which is a measure of instability, averaged across all subjects.

6. Calculate $\bar{D}(\Lambda)=\min _{\Lambda}\{\Lambda: \hat{D}(\Lambda) \leq \beta\}$ where we used $\beta=0.05$.

7. Select $\Lambda$ that yields the least amount of sparsity among all candidate regularization parameter sets with instability limited by $\beta$.

For selecting the number of clusters, we used a gap statistic as proposed by Tibshirani et al. [2001]. Generally, the gap statistic measures the within-cluster dispersion of a clustering of subjects given a certain number of clusters. The optimal number of clusters is then chosen as the smallest number that does not result in a significant increase in the gap statistic. A detailed description of calculating the gap statistic for our proposed RCCM is included in Appendix B.

\section{Simulations}

\subsection{Simulation Settings}

We conducted extensive simulations to examine the performance of our proposed RCCM, considering two different numbers of clusters, two different levels of magnitude of the precision matrix entries, and three different levels of similarity between the clusters. Specifically, data were generated 
with either $G=2$ clusters containing 67 and 37 subjects in each group, or $G=3$ clusters with 61,24 , and 19 subjects in each group which reflect the real data clustering results to be presented in Section 4. These data were generated for either a high or low magnitude setting, where the high magnitude setting corresponded to the off-diagonal entries of the true precision matrix having roughly three times the magnitude of the low magnitude setting entries on average. This introduced more distinction in precision matrices between clusters compared to the low magnitude setting. For all settings, 100 data sets were generated using $\mathrm{R}$ consisting of $n=177$ observations of $p=10$ variables on each of the $K=104$ subjects [R Core Team, 2018]. Dimensions in terms of the number of subjects, variables, and observations were chosen to match our data analysis described in Section 4. True networks and precision matrices were generated in a hierarchical manner beginning with group-level networks and precision matrices and then subject-level networks and matrices. That is, we first randomly generated either $G=2$ or $G=3$ hub-type networks, given by $\left\{N_{g}\right\}_{g=1}^{G}$, each with $\lfloor\sqrt{p}\rfloor$ hubs and thus $E=p-\lfloor\sqrt{p}\rfloor$ edges, while simultaneously forcing the networks to share $s=\lfloor\rho \times E\rfloor$ edges. We considered $\rho \in\{0.20,0.50,0.80\}$ for three different levels of overlap across the $G$ groups. Note that $\rho$ represents the approximate proportion of edges that are common across the cluster-level networks, and that these group-level networks were constant across the 100 simulations for a given $\rho$. Then, for each simulation we generated the cluster-level precision matrices, $\left\{\Omega_{0 \mathrm{~g}}\right\}_{g=1}^{G}$, as follows:

For $g=1$ to $G$;

1. Begin with a $p \times p$ adjacency matrix with sparsity structure corresponding to $N_{g}$.

2. Randomly draw $E$ values from a uniform distribution with support on the interval $[-1,-0.50] \cup[0.50,1]$ for elements corresponding to edges in the network to obtain $\Omega_{0 \mathrm{~g}}$.

3. Set the diagonal entries of $\Omega_{0 \mathrm{~g}}$ to 1 .

4. If $\Omega_{0 \mathrm{~g}}$ is not positive definite, then divide each row by its number of non-zero elements.

The $s$ number of edges that were common across the groups were forced to have the same values in each $\Omega_{0 \mathrm{~g}}$.

For the $K=104$ subjects, we first randomly assigned them to the $G$ clusters. Subjectlevel networks were then generated by randomly selecting $\lfloor 0.20 \times E\rfloor$ node pairs to add or remove an edge from their corresponding group-level network. For subject-level precision matrices, denoted $\left\{\Omega_{\mathbf{k}}\right\}_{k=1}^{104}$, common entries for subject- and corresponding group-level matrices were set the same as the group-level matrix with random noise generated from a $\mathcal{N}\left(0,0.05^{2}\right)$ distribution added to nonzero entries. Entries for added subject-specific edges were generated from a uniform distribution with support on the interval $[-1,-0.50] \cup[0.50,1]$. As before, if the generated $\Omega_{\mathrm{k}}$ was not positive definite, then we divided each row by its number of non-zero elements. Lastly, $n=177$ observations were randomly generated for each subject from a $\mathcal{N}_{p}\left(\mathbf{0}, \Omega_{\mathbf{k}}{ }^{-1}\right)$ distribution and were then centered and scaled prior to analysis. For tuning parameter selection, we implemented our modified stARS algorithm and included results for 5-fold CV in Tables 6, 7, and 8 in Appendix C.

\subsection{Simulation Results}

We assessed the performance of RCCM in two aspects: clustering and network estimation. Since existing methods only conduct clustering and FC network estimation separately, we considered competitive methods using a 2-step approach. For one approach, we started by obtaining GLasso estimates for each subject, and then clustering subjects based on vectorizing these estimates using Kmeans clustering, calling this GLasso \& K-means [Friedman et al., 2007]. For the second approach, 
we first used the Ward clustering method described in Section 2.2.2, and then implemented the group graphical lasso (GGL) of Danaher et al. [2014] for network estimation within each cluster, referred to as Ward \& GGL [Murtagh and Legendre, 2014]. GGL uses an $\ell_{1}$ penalty to encourage a shared sparsity pattern across subjects, but not necessarily entries of the same magnitude. For all approaches considered, we implemented our modified stARS approach described in Section 2.3 for regularization selection. We note that the scale of what worked well for tuning parameters varied across the methods, so we tailored them accordingly.

To assess clustering performance, we calculated the rand index (RI) and adjusted rand index $\left(\mathrm{RI}_{\mathrm{adj}}\right)$ with values of 1 indicating perfect clustering of subjects for both metrics [Rand, 1971. Hubert and Arabie, 1985]. For RCCM, cluster memberships were determined by assigning each subject to the cluster with the highest posterior probability as described by the $\hat{w}_{g k}$ estimates. Clustering performances of RCCM, Ward \& GGL, and GLasso \& K-means averaged across 100 simulations are displayed in Table 1 . Overall, clustering accuracy across all methods tended to be better for $G=2$ rather than $G=3$ clusters, and better for the high magnitude setting compared to the low magnitude setting as expected. We believe the exceptionally poor performance of the competing methods in the low magnitude settings is likely due to the high-level of similarity between clusters not favoring two-step approaches which fail to extract useful information from subjects for clustering. For the low magnitude setting RCCM achieved at best an average RI $=0.997$ and $\mathrm{RI}_{\text {adj }}=0.995$ in the setting with $G=2$ clusters and only $20 \%$ of group edges overlapping, and at worst an average $\mathrm{RI}=0.901$ and $\mathrm{RI}_{\mathrm{adj}}=0.805$ in the setting with $G=3$ clusters and $50 \%$ of group edges overlapping. Generally, for the low magnitude settings RCCM performed the best in terms of clustering among the three methods considered. However, the Ward \& GGL and GLasso \& K-means performed more competitively in the high magnitude setting with both methods outperforming RCCM in the $G=3$ setting with $80 \%$ of overlapping edges across the clusters.

For edge detection pertaining to network estimation, we calculated the true positive rate (TPR) or recall, the false positive rate (FPR), and the precision or positive predictive value (PPV) for both the subject and cluster-level networks. Performance in terms of edge-detection averaged across 100 simulations for $G=2$ and 3 clusters are displayed in Tables 2 and 3 respectively using our modified stARS method with RCCM, Ward \& GGL and GLasso \& K-means. In all tables, subject-level metrics are denoted with a subscript $k$, and group-level metrics subscript $g$.

Overall, we observe from Tables 2 and 3 that all three methods achieved higher power for the high magnitude settings compared to the low magnitude settings as expected. Moreover, the three methods performed better in terms of power as the proportion of overlapping edges across the clusters decreased. For RCCM and Ward \& GGL, this is likely due to improved clustering since across-cluster variability becomes comparable to within-cluster variability. For GLasso \& K-means, we believe the improved power as overlap decreased is due to the true precision matrices having entries slightly higher in magnitude on average for lower overlap settings compared to higher ones. This is since less adjustments needed to be made to ensure the true precision matrices shared enough edges while still being positive definite.

For the low magnitude settings with $G=2$ clusters displayed in Table 2, all three methods attained FPR values close to 0, displaying how stARS yields very sparse estimates. However, RCCM was the only method to maintain a reasonable power, displaying the advantage of concurrent estimation and clustering as opposed to conducting them sequentially. By pooling information across subjects in each group, RCCM was able to detect non-zero entries that were low in magnitude while the competing approaches with stARS yielded too much penalization. As expected, GLasso 
\& K-means generally had the lowest power of the three methods, as it does not pool information across subjects in network estimation. Simulation results for 5-fold CV are included in Appendix C.

\section{Data Analysis}

We applied the proposed RCCM to a resting-state fMRI data set collected on 40 participants diagnosed with chronic schizophrenia, 21 participants diagnosed with first-episode schizophrenia, and 43 healthy controls using our modified stARS algorithm for tuning parameter selection. Restingstate fMRI data were collected during an approximately 6-minute period using a Siemens Trio 3T scanner (Erlangen, Germany) with the following acquisition parameters: gradient-echo echo-planar imaging 180 volumes, repetition time $=2 \mathrm{~s}$, echo time $=30 \mathrm{~ms}$, flip angle $=90^{\circ}, 34$ contiguous ACPC aligned axial slices, voxel size $=3.4 \times 3.4 \times 4.0 \mathrm{~mm}$, matrix $=64 \times 64 \times 34$ [Camchong et al., 2011]. Additionally, a field map acquisition was collected and used to correct images for geometric distortion due to magnetic field inhomogeneities (repetition time $=300 \mathrm{~ms}$, echo time $=$ $1.91 \mathrm{~ms} / 4.37 \mathrm{~ms}$, flip angle $=55^{\circ}$, voxel size $=3.4 \times 3.4 \times 4.0 \mathrm{~mm}$ ). Imaging data were preprocessed using the software tool FEAT [Woolrich et al., 2001]. The first 3 volumes were removed prior to analysis, leaving 177 volumes in total. After preprocessing, data from 10 ROIs within the superior and inferior parietal lobules were extracted using an atlas developed through a parcellation study by Mars et al. [2011].

Resulting clusters of subjects obtained using our RCCM are referred to as groups A and B for $G=2$ clusters and groups $\mathrm{C}, \mathrm{D}$, and $\mathrm{E}$ for $G=3$. As displayed in Table 4 , specifying $G=2$ clusters yielded 67 and 37 subjects in groups A and B respectively. Most participants diagnosed with schizophrenia were classified into group A with $66.7 \%$ of participants diagnosed with first-episode schizophrenia and $75.0 \%$ of participants diagnosed with chronic-episode schizophrenia, while only about half of healthy controls were in the same group. Specifying $G=3$ clusters yielded clusters with 61, 24, and 19 subjects in groups $\mathrm{C}, \mathrm{D}$, and $\mathrm{E}$ respectively. Most participants diagnosed with schizophrenia were clustered into group C, with $57.1 \%$ of participants diagnosed with first-episode schizophrenia and $70.0 \%$ of participants diagnosed with chronic-episode schizophrenia respectively, and about half of the healthy controls in the same cluster. Applying the competing methods to our data, we found somewhat similar results as that of RCCM. In terms of clustering, the estimated cluster memberships for RCCM and Ward clustering had rand indexes of 0.873 and 0.891 when specifying $G=2$ and 3 clusters respectively, indicating that RCCM and Ward clustering agreed on roughly $90 \%$ of subject pairings. The estimated cluster memberships for GLasso \& K-means were less similar to that of RCCM, with rand indexes of 0.675 and 0.673 when specifying $G=2$ and 3 clusters respectively, indicating that RCCM and the GLasso \& K-means approach agreed on only about $70 \%$ of subject pairings.

Using a gap statistic as described in Appendix B, we selected $G=3$ as our final number of clusters [Tibshirani et al., 2001]. Generally, the gap statistic compares the observed change in within-cluster dispersion of a clustering of subjects when specifying different numbers of clusters to what is expected under a corresponding null setting. The performance of using a gap statistic for the proposed RCCM was investigated via simulations with the results summarized in Table 5$]$ of Appendix $\mathrm{C}$ as well.

Specifying $G=3$ clusters based on the results of the gap statistic, we observe that group $\mathrm{C}$ remained mostly the same as group A, but group B was further divided into groups D and E, suggesting more heterogeneity among subject-level estimates in group B compared to group A. This within-cluster heterogeneity in terms of edge presence is displayed in Figure 2, Overall, subjects were somewhat similar within each cluster, always having precision matrix entries with 
the same sign and similar in magnitude. However, not all implied networks were identical. This individual heterogeneity is displayed in Figure 2 showing the variance in edge presence calculated as $V_{i j}=p_{i j} \times\left(1-p_{i j}\right)$ where $p_{i j}$ is the proportion of subjects within each cluster with an edge present between variables $i$ and $j$.

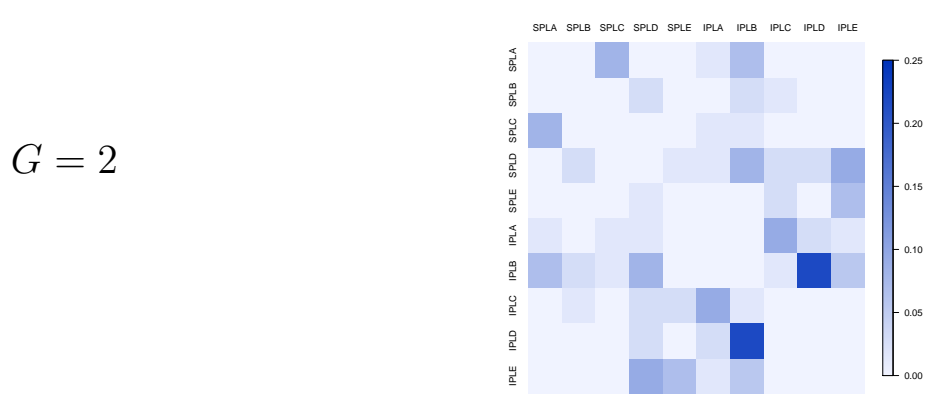

(A)

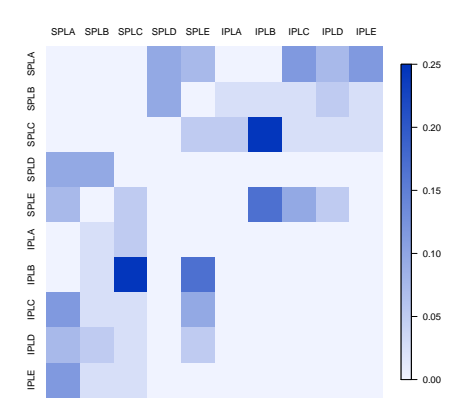

(B)

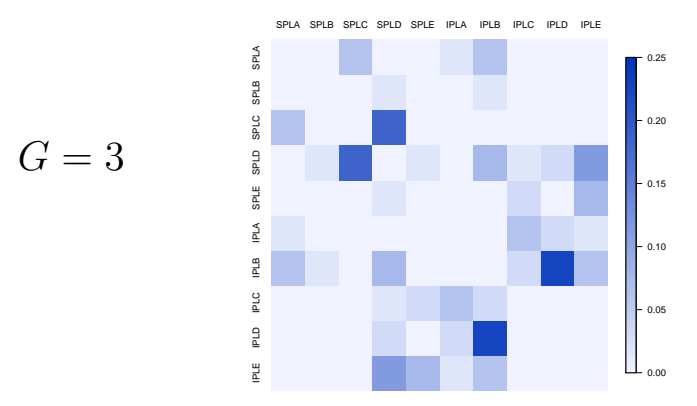

(C)

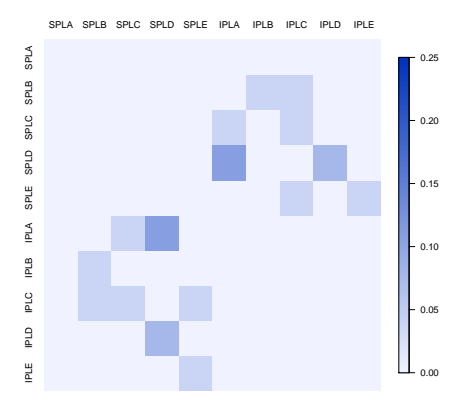

(D)

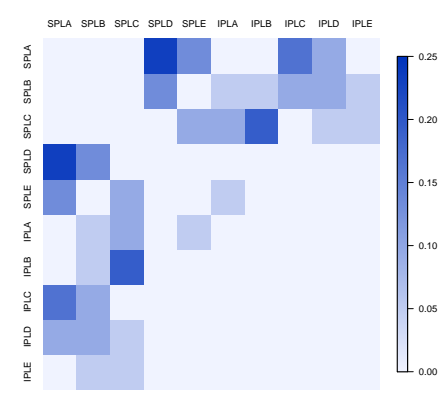

(E)

Figure 2: Variability in edge presence for groups A through E. A value of 0 indicates perfect agreement in edge presence within a cluster, while a value closer to 0.25 indicates a larger amount of heterogeneity in the presence of edges. Regions within the inferior parietal lobule are prefixed with IPL, while regions within the superior parietal lobule are prefixed with SPL.

Specifying either number of clusters, there was a tendency for participants diagnosed with schizophrenia to be clustered together more than healthy controls, but not significantly so. It is possible that subject-level heterogeneity in hemodynamic responses prevented better discrimination between those with schizophrenia and healthy controls. Moreover, it is unreasonable to expect perfect discrimination of participants since psychiatric disorders such as schizophrenia are complex and may not always yield consistent patterns in fMRI data across individuals [Wager and Lindquist, 2015].

Plots of estimated networks made using the igraph R package [Gabor and Tamas, 2006] are displayed in Figure 3. Regions within the inferior parietal lobule are prefixed with IPL, while regions within the superior parietal lobule are prefixed with SPL. Specifying $G=3$ clusters, we observe that the estimated group-level networks were somewhat similar, with all edges for group $\mathrm{C}$, the majority schizophrenia group, also being present for groups D and E which had significantly fewer participants diagnosed with schizophrenia. Moreover, estimated precision matrices were also similar in that common non-zero entries had the same sign. For example, the SPLA and SPLC nodes were connected in all three networks, and all had negative entries in their corresponding precision matrix estimates. However, it is notable that group C, the majority schizophrenia group, had fewer 
estimated connections than groups D and E. This is consistent with others who have found evidence supporting a general disconnection hypothesis in schizophrenia based on disrupted or decreased FC [Honey et al., 2005, Zhou et al., 2008, Yoon et al., 2008, Chen et al., 2018, Lottman et al., 2019].

$G=2$

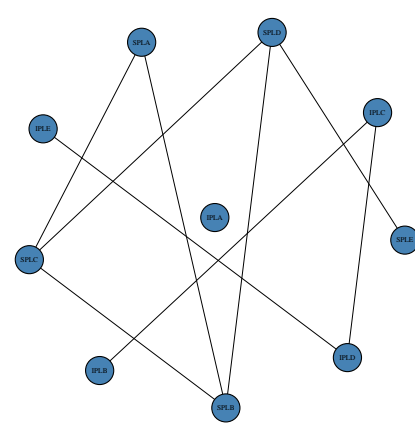

(A)

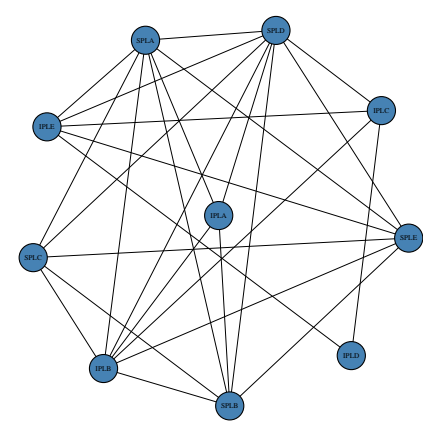

(B)

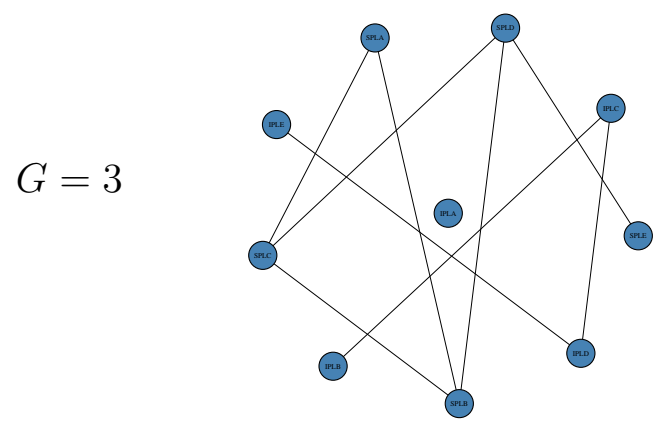

(C)

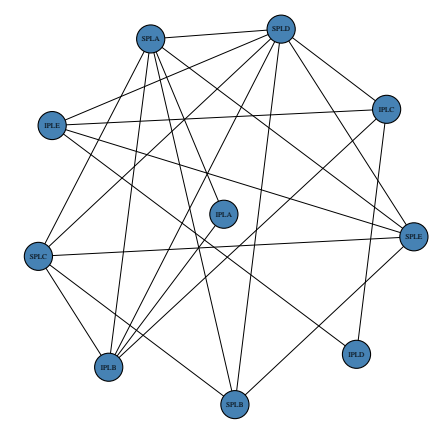

(D)

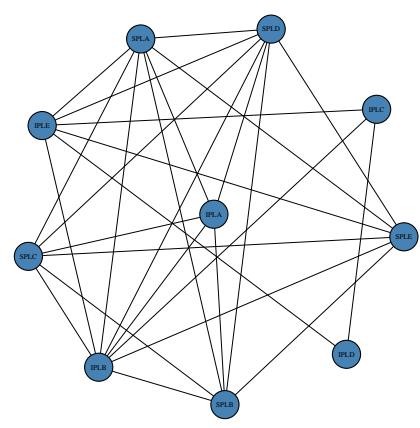

(E)

Figure 3: Estimated FC networks of the inferior and superior parietal lobules obtained using RCCM specifying $G=2$ or 3 clusters for the top and bottom rows respectively. For $G=2$ clusters, Group A contained the highest proportion of participants diagnosed with schizophrenia, and its estimated network had far fewer connections than Group B (top right) suggesting decreased FC among participants diagnosed with schizophrenia. For $G=3$ groups, Group C contained the majority of participants diagnosed with schizophrenia, and similarly had decreased FC relative to Groups D and E. Notably, Groups D and E were mostly made up of subjects belonging to Group B in the $G=2$ setting.

\section{Discussion}

Estimating FC for multi-subject fMRI data to better understand psychiatric disorders and neurodegenerative diseases is of clinical importance. We proposed a penalized model-based clustering method, the RCCM, to use fMRI data to simultaneously cluster subjects and provide interpretable estimates of both subject- and cluster-level FC networks. Additionally, we also proposed a modified stARS method for regularization selection that obtains more interpretable estimates of FC networks than current approaches. We showed the competitive performance of RCCM compared to conducting clustering and estimation sequentially rather than concurrently through simulations under varying settings. Lastly, we displayed the utility of RCCM through application to a resting-state fMRI data set collected on participants diagnosed with schizophrenia and healthy controls finding evidence to support the disconnection hypothesis among those diagnosed with schizophrenia. 
Although the proposed RCCM addresses short comings in current methods for analyzing multi-subject fMRI data sets, the method could be improved to better facilitate analyses in higher dimensions. Additionally, the proposed RCCM relies on the assumption of independent observations within subjects which is likely violated for fMRI data in which observations are known to exhibit autocorrelation. A model that explicitly accounts for this autocorrelation could be an extension of this work.

\section{Funding}

This work was supported by National Institutes of Health grant [1R03MH115300]; and Grant-in-Aid of Research, Artistry and Scholarship provided by University of Minnesota (to L. Z., A. D., and K. Q.). The content is solely the responsibility of the authors and does not necessarily represent the official views of the National Institutes of Health or the University of Minnesota.

\section{Acknowledgments}

Conflict of Interest: None declared.

\section{References}

J. I. Arribas, V. D. Calhoun, and T. Adali. Automatic bayesian classification of healthy controls, bipolar disorder, and schizophrenia using intrinsic connectivity maps from fmri data. IEEE Transactions on Biomedical Engineering, 57(12):2850-2860, 2010. ISSN 0018-9294.

J. Bien and R. Tibshirani. Sparse estimation of a covariance matrix. Biometrika, 98(4):807-820, 2011.

T. Cai, H. Li, W. Liu, and J. Xie. Joint estimation of multiple high-dimensional precision matrices. Statistica Sinica, 26(2):445-464, 2016. ISSN 10170405.

V. D. Calhoun, P. K. Maciejewski, G. D. Pearlson, and K. A. Kiehl. Temporal lobe and "default" hemodynamic brain modes discriminate between schizophrenia and bipolar disorder. Human Brain Mapping, 29(11):1265, 2008. ISSN 1097-0193.

J. Camchong, A. W. MacDonald, C. Bell, B. A. Mueller, and K. O. Lim. Altered functional and anatomical connectivity in schizophrenia. Schizophrenia Bulletin, 37(3):640-650, 2011. ISSN 0586-7614.

E. Castro, V. Gómez-Verdejo, M. Martínez-Ramón, K. A. Kiehl, and V. D. Calhoun. A multiple kernel learning approach to perform classification of groups from complex-valued fmri data analysis: Application to schizophrenia. NeuroImage, 87:1-17, 2014. ISSN 1053-8119.

S. Chen, Y. Xing, J. Kang, P. Kochunov, and L. E. Hong. Bayesian modeling of dependence in brain connectivity data. Biostatistics, 2018. ISSN 14654644.

P. Danaher, P. Wang, and D. M. Witten. The joint graphical lasso for inverse covariance estimation across multiple classes. Journal of the Royal Statistical Society, 76(2):373-397, 2014. ISSN 1369-7412.

C. Davatzikos, K. Ruparel, Y. Fan, D. G. Shen, M. Acharyya, J. W. Loughead, R. C. Gur, and D. D. Langleben. Classifying spatial patterns of brain activity with machine learning methods: Application to lie detection. Neuroimage, 28(3):663-668, 2005. ISSN 1053-8119.

A. P. Dempster, N. M. Laird, and D. B. Rubin. Maximum likelihood from incomplete data via the em algorithm. Journal of the Royal Statistical Society, 39(1):1-22, 1977. ISSN 0035-9246. 
E. Dennis and P. Thompson. Functional brain connectivity using fmri in aging and alzheimer's disease. Neuropsychology Review, 24(1):49-62, 2014. ISSN 1040-7308.

J. Fan, H. Liu, W. Wang, and Z. Zhu. Heterogeneity adjustment with applications to graphical model inference. Electronic Journal of Statistics, 12(2):3908-3952, 2018. ISSN 1935-7524.

M. Fiecas, I. Cribben, R. Bahktiari, and J. Cummine. A variance components model for statistical inference on functional connectivity networks. NeuroImage, 149:256-266, 2017. ISSN 10538119.

A. Fornito, A. Zalesky, C. Pantelis, and E. T. Bullmore. Schizophrenia, neuroimaging and connectomics. Neuroimage, 62(4):2296-2314, 2012. ISSN 1053-8119.

J. Friedman, T. Hastie, and R. Tibshirani. Sparse inverse covariance estimation with the graphical lasso. Biostatistics, 9(3):432-441, dec 2007. doi: 10.1093/biostatistics/kxm045.

K. J. Friston, C. D. Frith, P. F. Liddle, and R. S. J. Frackowiak. Functional connectivity: The principal-component analysis of large (pet) data sets. Journal of Cerebral Blood Flow \& Metabolism, 13(1):5-14, 1993. ISSN 0271-678X.

C. Gabor and N. Tamas. The igraph software package for complex network research. InterJournal, Complex Systems:1695, 2006. URL http://igraph.org.

C. Gao, Y. Zhu, X. Shen, and W. Pan. Estimation of multiple networks in gaussian mixture models. Electronic Journal of Statistics, 10(1), 2016. ISSN 1935-7524.

P. J. Green. On use of the em for penalized likelihood estimation. Journal of the Royal Statistical Society. Series B (Methodological), 52(3):443-452, 1990. ISSN 00359246.

J. Guo, E. Levina, G. Michailidis, and J. Zhu. Joint estimation of multiple graphical models. Biometrika, 98(1):1-15, 2011. ISSN 0006-3444.

B. Hao, W. W. Sun, Y. Liu, and G. Cheng. Simultaneous clustering and estimation of heterogeneous graphical models. Journal of Machine Learning Research, 18:1-58, 2018. ISSN 15324435.

S. M. Hill and S. Mukherjee. Network-based clustering with mixtures of 11-penalized gaussian graphical models: an empirical investigation, 2013.

G. D. Honey, E. Pomarol-Clotet, P. R. Corlett, R. A. E. Honey, P. J. Mckenna, E. T. Bullmore, and P. C. Fletcher. Functional dysconnectivity in schizophrenia associated with attentional modulation of motor function. Brain, 128(11):2597-2611, 2005. ISSN 0006-8950.

L. Hubert and P. Arabie. Comparing partitions. Journal of Classification, 2(1):193-218, 1985. ISSN 0176-4268.

S. L. Lauritzen. Graphical models. Oxford statistical science series ; 17. Clarendon Press ; Oxford University Press, Oxford : New York, repr. with corrections.. edition, 2004. ISBN 0198522193.

M. H. Lee, C. D. Smyser, and J. S. Shimony. Resting-state fmri: a review of methods and clinical applications. American Journal of Neuroradiology, 34(10):1866, 2013. ISSN 01956108.

H. Liu, K. Roeder, and L. Wasserman. Stability approach to regularization selection (stars) for high dimensional graphical models. In Proceedings of the 23rd International Conference on Neural Information Processing Systems - Volume 2, NIPS'10, pages 1432-1440, USA, 2010. Curran Associates Inc. URL http://dl .acm.org/citation. cfm?id=2997046.2997056.

K. K. Lottman, T. J. Gawne, N. V. Kraguljac, J. F. Killen, M. A. Reid, and A. C. Lahti. Examining resting-state functional connectivity in first-episode schizophrenia with $7 \mathrm{t} \mathrm{fmri}$ and meg. NeuroImage: Clinical, 24:101959, 2019. ISSN 2213-1582. 
R. B. Mars, S. Jbabdi, J. Sallet, J. X. O’Reilly, P. L. Croxson, E. Olivier, M. P. Noonan, C. Bergmann, A. S. Mitchell, M. G. Baxter, T. E. J. Behrens, H. Johansen-Berg, V. Tomassini, K. L. Miller, and M. F. S. Rushworth. Diffusion-weighted imaging tractography-based parcellation of the human parietal cortex and comparison with human and macaque resting-state functional connectivity. The Journal of Neuroscience, 31(11), 2011. ISSN 02706474.

X. Meng and D. B. Rubin. Maximum likelihood estimation via the ecm algorithm: A general framework. Biometrika, 80(2):267, 1993. doi: 10.2307/2337198.

R. S. Menon, S. Ogawa, S. G. Kim, J. M. Ellermann, H. Merkle, D. W. Tank, and K. Ugurbil. Functional brain mapping using magnetic resonance imaging. signal changes accompanying visual stimulation. Investigative Radiology, 27 Suppl 2:S47, 1992. ISSN 0020-9996.

S. Mueller, D. Wang, M. D. Fox, B. T. T. Yeo, J. Sepulcre, M. R. Sabuncu, R. Shafee, J. Lu, and H. Liu. Individual variability in functional connectivity architecture of the human brain. Neuron, 77(3):586-595, 2013. ISSN 0896-6273.

F. Murtagh and P. Legendre. Ward's hierarchical agglomerative clustering method: Which algorithms implement ward's criterion? Journal of Classification, 31(3):274-295, 2014. ISSN 0176-4268.

W. Pettersson-Yeo, P. Allen, S. Benetti, P. Mcguire, and A. Mechelli. Dysconnectivity in schizophrenia: Where are we now? Neuroscience and Biobehavioral Reviews, 35(5):1110-1124, 2011. ISSN 0149-7634.

R. B. Price, S. Lane, K. Gates, T. E. Kraynak, M. S. Horner, M. E. Thase, and G. J. Siegle. Parsing heterogeneity in the brain connectivity of depressed and healthy adults during positive mood. Biological Psychiatry, 81(4):347-357, 2017. ISSN 0006-3223.

H. Qiu, F. Han, H. Liu, and B. Caffo. Joint estimation of multiple graphical models from high dimensional time series. Journal of the Royal Statistical Society, 78(2):487-504, 2016. ISSN 13697412.

R Core Team. R: A Language and Environment for Statistical Computing. R Foundation for Statistical Computing, Vienna, Austria, 2018. URL https://www.R-project.org/.

W. M. Rand. Objective criteria for the evaluation of clustering methods. Journal of the American Statistical Association, 66(336):846-850, 1971. ISSN 0162-1459.

H. Shen, L. Wang, Y. Liu, and D. Hu. Discriminative analysis of resting-state functional connectivity patterns of schizophrenia using low dimensional embedding of fmri. NeuroImage , 49(4):31103121, 2010. ISSN 1053-8119.

K. A. Smitha, K. A. Raja, K. M. Arun, P. G. Rajesh, B. Thomas, T. R. Kapilamoorthy, and C. Kesavadas. Resting state fmri: A review on methods in resting state connectivity analysis and resting state networks. The Neuroradiology Journal, 30(4):305-317, 2017. ISSN 1971-4009.

Tibshirani, R., Walther, G. and Hastie, T. Estimating the number of clusters in a data set via the gap statistic. Journal of the Royal Statistical Society. Series B, Statistical Methodology, 63(2): 411-423, 2001.

T. D. Wager and M. A. Lindquist. Principles of fMRI, chapter 6. Leanpub, 2015.

M. W. Woolrich, B. D. Ripley, M. Brady, and S. M. Smith. Temporal autocorrelation in univariate linear modeling of fmri data. NeuroImage, 14(6):1370-1386, 2001. ISSN 1053-8119.

J. H. Yoon, M. J. Minzenberg, S. Ursu, R. Walters, C. Wendelken, J. D. Ragland, and C. S. Carter. Association of dorsolateral prefrontal cortex dysfunction with disrupted coordinated brain activity 
in schizophrenia: Relationship with impaired cognition, behavioral disorganization, and global function. American Journal of Psychiatry, 165(8):1006-1014, 2008. ISSN 0002-953X.

L. Zeng, H. Shen, L. Liu, and D. Hu. Unsupervised classification of major depression using functional connectivity mri. Human Brain Mapping, 35(4):1630-1641, 2014. ISSN 1065-9471.

L. Zhang, A. DiLernia, K. Quevedo, J. Camchong, K. Lim, and W. Pan. A random covariance model for bi-level graphical modeling with application to resting-state fmri data, Biometrics, 2020.

H. Zhou, W. Pan, and X. Shen. Penalized model-based clustering with unconstrained covariance matrices. Electronic Journal of Statistics, 3(1473-1496):1473-1496, 2009. ISSN 1935-7524.

Y. Zhou, N. Shu, Y. Liu, M. Song, Y. Hao, H. Liu, C. Yu, Z. Liu, and T. Jiang. Altered restingstate functional connectivity and anatomical connectivity of hippocampus in schizophrenia. Schizophrenia Research, 100(1-3):120-132, 2008. ISSN 0920-9964.

X. Zhu, X. Wang, J. Xiao, J. Liao, M. Zhong, W. Wang, and S. Yao. Evidence of a dissociation pattern in resting-state default mode network connectivity in first-episode, treatment-naive major depression patients. Biological Psychiatry, 71(7):611-617, 2012. ISSN 0006-3223.

Y. Zhu, X. Shen, and W. Pan. Structural pursuit over multiple undirected graphs. Journal of the American Statistical Association, 109(508):00-00, 2014. ISSN 0162-1459. 
Table 1: Clustering performance of RCCM, Ward, and K-means clustering using stARS for tuning parameter selection. Results are for $G=2$ and 3 unbalanced groups, true precision matrices with entries large or small in magnitude, and group overlap being $0.20,0.50$, or 0.80 for each of 104 subjects averaged across 100 simulations. Ward clustering was based on a difference matrix of the Frobenius-norm differences between matrix estimates, while K-means clustering was based on vectorized GLasso matrix estimates.

\begin{tabular}{|c|c|c|c|c|c|}
\hline $\bar{G}$ & Magnitude & Overlap & Method & RI & $\mathrm{RI}_{\mathrm{adj}}$ \\
\hline \multirow[t]{18}{*}{2} & \multirow{9}{*}{ High } & \multirow[t]{3}{*}{0.2} & $\mathrm{RCCM}$ & $1.000(0.000)$ & $1.000(0.000)$ \\
\hline & & & Ward \& GGL & $1.000(0.000)$ & $1.000(0.000)$ \\
\hline & & & GLasso \& K-means & $1.000(0.000)$ & $1.000(0.000)$ \\
\hline & & \multirow[t]{3}{*}{0.5} & RCCM & $0.995(0.046)$ & $0.990(0.100)$ \\
\hline & & & Ward \& GGL & $1.000(0.000)$ & $1.000(0.000)$ \\
\hline & & & GLasso \& K-means & $0.987(0.072)$ & $0.975(0.145)$ \\
\hline & & \multirow[t]{3}{*}{0.8} & RCCM & $1.000(0.000)$ & $1.000(0.000)$ \\
\hline & & & Ward \& GGL & $1.000(0.000)$ & $1.000(0.000)$ \\
\hline & & & GLasso \& K-means & $1.000(0.000)$ & $1.000(0.000)$ \\
\hline & \multirow[t]{9}{*}{ Low } & \multirow[t]{3}{*}{0.2} & $\mathrm{RCCM}$ & $0.997(0.008)$ & $0.995(0.015)$ \\
\hline & & & Ward \& GGL & $0.971(0.031)$ & $0.942(0.062)$ \\
\hline & & & GLasso \& K-means & $0.529(0.013)$ & $-0.007(0.023)$ \\
\hline & & \multirow[t]{3}{*}{0.5} & RCCM & $0.993(0.012)$ & $0.986(0.023)$ \\
\hline & & & Ward \& GGL & $0.962(0.038)$ & $0.923(0.076)$ \\
\hline & & & GLasso \& K-means & $0.531(0.031)$ & $-0.002(0.066)$ \\
\hline & & \multirow[t]{3}{*}{0.8} & $\mathrm{RCCM}$ & $0.982(0.030)$ & $0.963(0.060)$ \\
\hline & & & Ward \& GGL & $0.921(0.067)$ & $0.842(0.135)$ \\
\hline & & & GLasso \& K-means & $0.527(0.012)$ & $-0.008(0.024)$ \\
\hline \multirow[t]{18}{*}{3} & \multirow[t]{9}{*}{ High } & \multirow[t]{3}{*}{0.2} & $\mathrm{RCCM}$ & $1.000(0.000)$ & $1.000(0.000)$ \\
\hline & & & Ward \& GGL & $1.000(0.000)$ & $1.000(0.000)$ \\
\hline & & & GLasso \& K-means & $0.865(0.125)$ & $0.717(0.263)$ \\
\hline & & \multirow[t]{3}{*}{0.5} & RCCM & $1.000(0.000)$ & $1.000(0.000)$ \\
\hline & & & Ward \& GGL & $1.000(0.000)$ & $1.000(0.000)$ \\
\hline & & & GLasso \& K-means & $0.888(0.127)$ & $0.765(0.266)$ \\
\hline & & \multirow[t]{3}{*}{0.8} & $\mathrm{RCCM}$ & $0.878(0.116)$ & $0.767(0.219)$ \\
\hline & & & Ward \& GGL & $0.999(0.003)$ & $0.998(0.007)$ \\
\hline & & & GLasso \& K-means & $0.975(0.077)$ & $0.948(0.156)$ \\
\hline & \multirow[t]{9}{*}{ Low } & \multirow[t]{3}{*}{0.2} & RCCM & $0.910(0.058)$ & $0.822(0.112)$ \\
\hline & & & Ward \& GGL & $0.892(0.060)$ & $0.786(0.117)$ \\
\hline & & & GLasso \& K-means & $0.526(0.031)$ & $-0.002(0.066)$ \\
\hline & & \multirow[t]{3}{*}{0.5} & $\mathrm{RCCM}$ & $0.901(0.062)$ & $0.805(0.120)$ \\
\hline & & & Ward \& GGL & $0.872(0.067)$ & $0.748(0.132)$ \\
\hline & & & GLasso \& K-means & $0.513(0.011)$ & $-0.030(0.019)$ \\
\hline & & \multirow[t]{3}{*}{0.8} & RCCM & $0.917(0.058)$ & $0.835(0.119)$ \\
\hline & & & Ward \& GGL & $0.885(0.061)$ & $0.771(0.121)$ \\
\hline & & & GLasso \& K-means & $0.520(0.015)$ & $-0.013(0.027)$ \\
\hline
\end{tabular}


Table 2: Comparison of method performances for $G=2$ unbalanced groups containing 67 and 37 subjects. Results are for observations of $p=10$ variables and $n=177$ observations for each subject using stARs for tuning parameter selection, averaged across 100 simulations. Grouplevel performance measures for GLasso are missing since the method does not yield cluster-level estimates.

\begin{tabular}{|c|c|c|c|c|c|c|c|c|}
\hline Magnitude & Overlap & Method & $\mathrm{TPR}_{\mathrm{g}}$ & $\mathrm{FPR}_{\mathrm{g}}$ & $\mathrm{PPV}_{\mathrm{g}}$ & $\mathrm{TPR}_{\mathrm{k}}$ & $\mathrm{FPR}_{\mathrm{k}}$ & $\mathrm{PPV}_{\mathrm{k}}$ \\
\hline \multirow[t]{18}{*}{ High } & \multirow[t]{6}{*}{0.2} & \multirow[t]{2}{*}{ RCCM } & 0.913 & 0.091 & 0.673 & 1.000 & 0.134 & 0.580 \\
\hline & & & $(0.000)$ & $(0.006)$ & $(0.014)$ & $(0.000)$ & $(0.004)$ & $(0.008)$ \\
\hline & & \multirow[t]{2}{*}{ Ward \& GGL } & 0.913 & 0.088 & 0.682 & 1.000 & 0.092 & 0.666 \\
\hline & & & $(0.000)$ & $(0.006)$ & $(0.015)$ & $(0.000)$ & $(0.003)$ & $(0.007)$ \\
\hline & & \multirow{2}{*}{\multicolumn{2}{|c|}{ GLasso \& K-means }} & & & 1.000 & 0.093 & 0.664 \\
\hline & & & & & & $(0.000)$ & $(0.003)$ & $(0.007)$ \\
\hline & \multirow[t]{6}{*}{0.5} & \multirow[t]{2}{*}{ RCCM } & 1.000 & 0.066 & 0.724 & 0.929 & 0.120 & 0.587 \\
\hline & & & $(0.000)$ & $(0.005)$ & $(0.017)$ & $(0.004)$ & $(0.006)$ & $(0.012)$ \\
\hline & & \multirow[t]{2}{*}{ Ward \& GGL } & 1.000 & 0.084 & 0.675 & 0.921 & 0.076 & 0.689 \\
\hline & & & $(0.000)$ & $(0.005)$ & $(0.013)$ & $(0.002)$ & $(0.003)$ & $(0.008)$ \\
\hline & & \multirow{2}{*}{ GLasso \& K-means } & & & & 0.921 & 0.077 & 0.687 \\
\hline & & & & & & $(0.002)$ & $(0.003)$ & $(0.009)$ \\
\hline & \multirow[t]{6}{*}{0.8} & \multirow[t]{2}{*}{ RCCM } & 0.705 & 0.078 & 0.710 & 0.910 & 0.148 & 0.641 \\
\hline & & & $(0.155)$ & $(0.025)$ & $(0.094)$ & $(0.033)$ & $(0.009)$ & $(0.028)$ \\
\hline & & \multirow{2}{*}{ Ward \& GGL } & 0.900 & 0.045 & 0.847 & 0.848 & 0.093 & 0.726 \\
\hline & & & $(0.051)$ & $(0.024)$ & $(0.075)$ & $(0.066)$ & $(0.018)$ & $(0.050)$ \\
\hline & & \multirow[t]{2}{*}{ GLasso \& K-means } & & & & 0.856 & 0.091 & 0.731 \\
\hline & & & & & & $(0.054)$ & $(0.014)$ & $(0.038)$ \\
\hline \multirow[t]{18}{*}{ Low } & \multirow[t]{6}{*}{0.2} & \multirow[t]{2}{*}{ RCCM } & 0.588 & 0.120 & 0.507 & 0.887 & 0.011 & 0.947 \\
\hline & & & $(0.099)$ & $(0.036)$ & $(0.093)$ & $(0.037)$ & $(0.005)$ & $(0.021)$ \\
\hline & & \multirow[t]{2}{*}{ Ward \& GGL } & 0.091 & 0.018 & 0.522 & 0.238 & 0.002 & 0.965 \\
\hline & & & $(0.059)$ & $(0.013)$ & $(0.282)$ & $(0.087)$ & $(0.002)$ & $(0.030)$ \\
\hline & & \multirow[t]{2}{*}{ GLasso \& K-means } & & & & 0.124 & 0.000 & 0.994 \\
\hline & & & & & & $(0.044)$ & $(0.001)$ & $(0.015)$ \\
\hline & \multirow[t]{6}{*}{0.5} & \multirow[t]{2}{*}{$\mathrm{RCCM}$} & 0.592 & 0.148 & 0.524 & 0.806 & 0.020 & 0.924 \\
\hline & & & $(0.095)$ & $(0.050)$ & $(0.092)$ & $(0.063)$ & $(0.007)$ & $(0.024)$ \\
\hline & & \multirow[t]{2}{*}{ Ward \& GGL } & 0.096 & 0.020 & 0.644 & 0.155 & 0.002 & 0.961 \\
\hline & & & $(0.072)$ & $(0.028)$ & $(0.279)$ & $(0.067)$ & $(0.002)$ & $(0.037)$ \\
\hline & & \multirow[t]{2}{*}{ GLasso \& K-means } & & & & 0.159 & 0.000 & 0.993 \\
\hline & & & & & & $(0.019)$ & $(0.000)$ & $(0.007)$ \\
\hline & \multirow[t]{6}{*}{0.8} & \multirow[t]{2}{*}{$\mathrm{RCCM}$} & 0.574 & 0.115 & 0.650 & 0.639 & 0.017 & 0.936 \\
\hline & & & $(0.125)$ & $(0.076)$ & $(0.081)$ & $(0.136)$ & $(0.006)$ & $(0.017)$ \\
\hline & & \multirow[t]{2}{*}{ Ward \& GGL } & 0.097 & 0.015 & 0.778 & 0.130 & 0.001 & 0.977 \\
\hline & & & $(0.059)$ & $(0.025)$ & $(0.235)$ & $(0.048)$ & $(0.001)$ & $(0.023)$ \\
\hline & & \multirow[t]{2}{*}{ GLasso \& K-means } & & & & 0.118 & 0.000 & 0.993 \\
\hline & & & & & & $(0.017)$ & $(0.000)$ & $(0.006)$ \\
\hline
\end{tabular}


Table 3: Comparison of method performances for $G=3$ unbalanced groups containing 61, 24, and 19 subjects. Results are for observations of $p=10$ variables and $n=177$ observations for each subject using stARs for tuning parameter selection, averaged across 100 simulations. Grouplevel performance measures for GLasso are missing since the method does not yield cluster-level estimates.

\begin{tabular}{|c|c|c|c|c|c|c|c|c|}
\hline Magnitude & Overlap & Method & $\mathrm{TPR}_{\mathrm{g}}$ & $\mathrm{FPR}_{\mathrm{g}}$ & $\mathrm{PPV}_{\mathrm{g}}$ & $\mathrm{TPR}_{\mathrm{k}}$ & $\mathrm{FPR}_{\mathrm{k}}$ & $\mathrm{PPV}_{\mathrm{k}}$ \\
\hline \multirow[t]{18}{*}{ High } & \multirow[t]{6}{*}{0.2} & \multirow[t]{2}{*}{ RCCM } & 0.999 & 0.143 & 0.598 & 0.997 & 0.159 & 0.570 \\
\hline & & & $(0.007)$ & $(0.021)$ & $(0.029)$ & $(0.008)$ & $(0.011)$ & $(0.009)$ \\
\hline & & \multirow[t]{2}{*}{ Ward \& GGL } & 0.998 & 0.108 & 0.663 & 0.988 & 0.120 & 0.636 \\
\hline & & & $(0.011)$ & $(0.016)$ & $(0.030)$ & $(0.010)$ & $(0.013)$ & $(0.017)$ \\
\hline & & \multirow[t]{2}{*}{ GLasso \& K-means } & & & & 0.988 & 0.120 & 0.636 \\
\hline & & & & & & $(0.010)$ & $(0.013)$ & $(0.016)$ \\
\hline & \multirow[t]{6}{*}{0.5} & \multirow[t]{2}{*}{ RCCM } & 0.994 & 0.133 & 0.653 & 0.999 & 0.170 & 0.596 \\
\hline & & & $(0.056)$ & $(0.022)$ & $(0.039)$ & $(0.009)$ & $(0.014)$ & $(0.028)$ \\
\hline & & \multirow[t]{2}{*}{ Ward \& GGL } & 1.000 & 0.086 & 0.745 & 0.997 & 0.124 & 0.667 \\
\hline & & & $(0.000)$ & $(0.017)$ & $(0.039)$ & $(0.002)$ & $(0.007)$ & $(0.012)$ \\
\hline & & \multirow[t]{2}{*}{ GLasso \& K-means } & & & & 0.977 & 0.120 & 0.678 \\
\hline & & & & & & $(0.116)$ & $(0.022)$ & $(0.056)$ \\
\hline & \multirow[t]{6}{*}{0.8} & \multirow[t]{2}{*}{ RCCM } & 0.869 & 0.113 & 0.673 & 0.969 & 0.152 & 0.615 \\
\hline & & & $(0.027)$ & $(0.002)$ & $(0.007)$ & $(0.006)$ & $(0.007)$ & $(0.010)$ \\
\hline & & \multirow[t]{2}{*}{ Ward \& GGL } & 0.858 & 0.093 & 0.714 & 0.913 & 0.094 & 0.709 \\
\hline & & & $(0.033)$ & $(0.015)$ & $(0.032)$ & $(0.012)$ & $(0.008)$ & $(0.017)$ \\
\hline & & \multirow[t]{2}{*}{ GLasso \& K-means } & & & & 0.914 & 0.095 & 0.708 \\
\hline & & & & & & $(0.011)$ & $(0.008)$ & $(0.016)$ \\
\hline \multirow[t]{18}{*}{ Low } & \multirow[t]{6}{*}{0.2} & \multirow[t]{2}{*}{ RCCM } & 0.984 & 0.020 & 0.912 & 0.935 & 0.017 & 0.926 \\
\hline & & & $(0.065)$ & $(0.019)$ & $(0.083)$ & $(0.040)$ & $(0.010)$ & $(0.040)$ \\
\hline & & \multirow[t]{2}{*}{ Ward \& GGL } & 0.298 & 0.000 & 0.994 & 0.314 & 0.002 & 0.976 \\
\hline & & & $(0.091)$ & $(0.004)$ & $(0.067)$ & $(0.073)$ & $(0.002)$ & $(0.021)$ \\
\hline & & \multirow[t]{2}{*}{ GLasso \& K-means } & & & & 0.158 & 0.000 & 0.996 \\
\hline & & & & & & $(0.020)$ & $(0.000)$ & $(0.005)$ \\
\hline & \multirow[t]{6}{*}{0.5} & \multirow[t]{2}{*}{$\mathrm{RCCM}$} & 0.801 & 0.005 & 0.974 & 0.796 & 0.006 & 0.975 \\
\hline & & & $(0.124)$ & $(0.024)$ & $(0.125)$ & $(0.041)$ & $(0.002)$ & $(0.008)$ \\
\hline & & \multirow[t]{2}{*}{ Ward \& GGL } & 0.192 & 0.002 & 0.966 & 0.222 & 0.002 & 0.966 \\
\hline & & & $(0.080)$ & $(0.009)$ & $(0.170)$ & $(0.062)$ & $(0.002)$ & $(0.032)$ \\
\hline & & \multirow[t]{2}{*}{ GLasso \& K-means } & & & & 0.107 & 0.001 & 0.990 \\
\hline & & & & & & $(0.041)$ & $(0.002)$ & $(0.024)$ \\
\hline & \multirow[t]{6}{*}{0.8} & \multirow[t]{2}{*}{ RCCM } & 0.746 & 0.028 & 0.897 & 0.702 & 0.028 & 0.899 \\
\hline & & & $(0.066)$ & $(0.025)$ & $(0.088)$ & $(0.051)$ & $(0.017)$ & $(0.052)$ \\
\hline & & \multirow[t]{2}{*}{ Ward \& GGL } & 0.232 & 0.001 & 0.992 & 0.274 & 0.006 & 0.946 \\
\hline & & & $(0.100)$ & $(0.005)$ & $(0.056)$ & $(0.077)$ & $(0.004)$ & $(0.027)$ \\
\hline & & \multirow[t]{2}{*}{ GLasso \& K-means } & & & & 0.162 & 0.001 & 0.991 \\
\hline & & & & & & $(0.022)$ & $(0.000)$ & $(0.007)$ \\
\hline
\end{tabular}


Table 4: Summary of clustering results for specifying $G=2$ or 3 clusters. For $G=2$, Group A had the highest proportion of participants diagnosed with schizophrenia with $66.7 \%$ of first-episode participants, and $75.0 \%$ of participants diagnosed with chronic-episode schizophrenia. Healthy controls, however, were more evenly distributed between the two groups, with $53.5 \%$ and $46.5 \%$ in Groups A and B respectively. Results for $G=3$ groups were not as clear, with most subjects being clustered into Group C.

\begin{tabular}{cclll}
\hline$G$ & Group & Control & 1st Episode & Chronic \\
\hline 2 & $\mathrm{~A}$ & $23(53.5 \%)$ & $14(66.7 \%)$ & $30(75.0 \%)$ \\
\cline { 2 - 5 } & $\mathrm{B}$ & $20(46.5 \%)$ & $7(33.3 \%)$ & $10(25.0 \%)$ \\
\hline 3 & $\mathrm{C}$ & $21(48.8 \%)$ & $12(57.1 \%)$ & $28(70.0 \%)$ \\
\cline { 2 - 5 } & $\mathrm{D}$ & $10(23.3 \%)$ & $6(28.6 \%)$ & $8(20.0 \%)$ \\
\cline { 2 - 5 } & $\mathrm{E}$ & $12(27.9 \%)$ & $3(14.3 \%)$ & $4(10.0 \%)$ \\
\hline
\end{tabular}




\section{Appendix A}

\section{Updates for $\pi_{\mathrm{g}}$ 's}

With a LaGrange multiplier constraint to force $\sum_{g=1}^{G} \pi_{g}=1$, the terms of $Q\left(\Theta ; \Theta^{(r)}\right)$ containing $\pi_{g}$ 's are

$$
Q_{\pi}\left(\Theta ; \Theta^{(r)}\right)=-2 \sum_{k=1}^{K} \sum_{g=1}^{G} w_{g k}^{(r)}\left(\log \pi_{g}\right)+\gamma\left(\sum_{g=1}^{G} \pi_{g}-1\right)
$$

so

$$
\begin{gathered}
\frac{\partial Q_{\pi}\left(\Theta ; \Theta^{(r)}\right)}{\partial \pi_{g}}=-\frac{2}{\pi_{g}} \sum_{k=1}^{K} w_{g k}^{(r)}+\gamma=0 \Longrightarrow \\
\pi_{g} \gamma=2 \sum_{k=1}^{K} w_{g k}^{(r)} \Longrightarrow \pi_{g}^{(r+1)}=\frac{1}{K} \sum_{k=1}^{K} w_{g k}^{(r)}
\end{gathered}
$$

since $\sum_{g=1}^{G} \pi_{g}=\sum_{g=1}^{G} w_{g k}^{(r)}=1$.

\section{Updates for $\Omega_{0 \mathrm{~g}}$ 's}

The terms of $Q\left(\Theta ; \Theta^{(r)}\right)$ containing $\Omega_{0 \mathrm{~g}}$ 's are

$$
\begin{aligned}
& Q_{\boldsymbol{\Omega}_{\mathbf{0 g}}}\left(\Theta ; \Theta^{(r)}\right)=-2 \sum_{k=1}^{K} w_{g k}^{(r)} \log \left(p_{g}\left(\boldsymbol{\Omega}_{\mathbf{k}}^{(r)} ; \lambda_{2}, \boldsymbol{\Omega}_{\mathbf{0 g}}\right)\right)+\lambda_{3}\left\|\boldsymbol{\Omega}_{\mathbf{0 g}}\right\|_{1}= \\
& -2 \sum_{k=1}^{K} w_{g k}^{(r)}\left(-\frac{\lambda_{2}}{2} \log \left|\frac{1}{\lambda_{2}} \boldsymbol{\Omega}_{\mathbf{0 g}}\right|-\frac{1}{2} \operatorname{tr}\left(\lambda_{2} \boldsymbol{\Omega}_{\mathbf{0 g}}{ }^{-1} \boldsymbol{\Omega}_{\mathbf{k}}{ }^{(r)}\right)\right)+\lambda_{3}|| \boldsymbol{\Omega}_{\mathbf{0 g}} \|_{1}= \\
& \lambda_{2} \sum_{k=1}^{K} w_{g k}^{(r)}\left(\log \left|\frac{1}{\lambda_{2}} \boldsymbol{\Omega}_{\mathbf{0 g}}\right|\right)+\lambda_{2} \sum_{k=1}^{K} w_{g k}^{(r)} \operatorname{tr}\left(\boldsymbol{\Omega}_{\mathbf{0 g}}{ }^{-1} \boldsymbol{\Omega}_{\mathbf{k}}{ }^{(r)}\right)+\lambda_{3}\left\|\boldsymbol{\Omega}_{\mathbf{0 g}}\right\|_{1}= \\
& \operatorname{tr}\left(\lambda_{2} \sum_{k=1}^{K} w_{g k}^{(r)} \mathbf{\Omega}_{\mathbf{k}}^{(r)} \boldsymbol{\Omega}_{\mathbf{0 g}}{ }^{-1}\right)+\lambda_{2} \sum_{k=1}^{K} w_{g k}^{(r)} \log \left|\boldsymbol{\Omega}_{\mathbf{0 g}}\right|+\lambda_{3}|| \boldsymbol{\Omega}_{\mathbf{0 g}} \|_{1} \propto \\
& \operatorname{tr}\left(\frac{\sum_{k=1}^{K} w_{g k}^{(r)} \boldsymbol{\Omega}_{\mathbf{k}}^{(r)}}{\sum_{k=1}^{K} w_{g k}^{(r)}} \boldsymbol{\Omega}_{\mathbf{0 g}}{ }^{-1}\right)+\log \left|\boldsymbol{\Omega}_{\mathbf{0 g}}\right|+\frac{\lambda_{3}}{\lambda_{2} \sum_{k=1}^{K} w_{g k}^{(r)}}\left\|\boldsymbol{\Omega}_{\mathbf{0 g}}\right\|_{1},
\end{aligned}
$$

which can be solved using the covariance lasso algorithm. 


\section{Updates for $\Omega_{\mathrm{k}}$ 's}

The terms of $Q\left(\Theta ; \Theta^{(r)}\right)$ containing $\Omega_{\mathbf{k}}$ 's are

$$
\begin{aligned}
& Q_{\Omega_{k}}\left(\Theta ; \Theta^{(r)}\right)=\sum_{t=1}^{n_{k}}\left(-\log \left|\boldsymbol{\Omega}_{\mathbf{k}}\right|+\mathbf{y}_{\mathbf{k t}}^{\mathbf{T}} \boldsymbol{\Omega}_{\mathbf{k}} \mathbf{y}_{\mathbf{k t}}\right)-2 \sum_{g=1}^{G} w_{g k}^{(r)}\left(\log p_{g}\left(\boldsymbol{\Omega}_{\mathbf{k}} ; \lambda_{2}, \boldsymbol{\Omega}_{\mathbf{0 g}}{ }^{(r)}\right)\right)+\lambda_{1}\left\|\boldsymbol{\Omega}_{\mathbf{k}}\right\|_{1}= \\
& \sum_{t=1}^{n_{k}}\left(\mathbf{y}_{\mathbf{k t}}^{\mathbf{T}} \boldsymbol{\Omega}_{\mathbf{k}} \mathbf{y}_{\mathbf{k t}}-\log \left|\boldsymbol{\Omega}_{\mathbf{k}}\right|\right)-2 \sum_{g=1}^{G} w_{g k}^{(r)}\left(\frac{\lambda_{2}-p-1}{2} \log \left|\boldsymbol{\Omega}_{\mathbf{k}}\right|-\frac{1}{2} \operatorname{tr}\left(\lambda_{2} \boldsymbol{\Omega}_{\mathbf{0 g}}{ }^{(r)^{-1}} \boldsymbol{\Omega}_{\mathbf{k}}\right)\right)+\lambda_{1}|| \boldsymbol{\Omega}_{\mathbf{k}} \|_{1}= \\
& \sum_{t=1}^{n_{k}}\left(\operatorname{tr}\left(\mathbf{y}_{\mathbf{k t}} \mathbf{y}_{\mathbf{k t}}^{\mathbf{T}} \boldsymbol{\Omega}_{\mathbf{k}}\right)-\log \left|\boldsymbol{\Omega}_{\mathbf{k}}\right|\right)+\sum_{g=1}^{G} w_{g k}^{(r)}\left(\lambda_{2} \operatorname{tr}\left(\boldsymbol{\Omega}_{\mathbf{0 g}}{ }^{(r)^{-1}} \boldsymbol{\Omega}_{\mathbf{k}}\right)-\left(\lambda_{2}-p-1\right) \log \left|\boldsymbol{\Omega}_{\mathbf{k}}\right|\right)+\lambda_{1}|| \boldsymbol{\Omega}_{\mathbf{k}} \|_{1}= \\
& \operatorname{tr}\left(n_{k} S_{k} \boldsymbol{\Omega}_{\mathbf{k}}\right)+\operatorname{tr}\left(\lambda_{2} \sum_{g=1}^{G} w_{g k}^{(r)} \boldsymbol{\Omega}_{\mathbf{0 g}}{ }^{(r)^{-1}} \boldsymbol{\Omega}_{\mathbf{k}}\right)-\log \left|\boldsymbol{\Omega}_{\mathbf{k}}\right|\left(n_{k}+\left(\lambda_{2}-p-1\right)\right)+\lambda_{1}|| \boldsymbol{\Omega}_{\mathbf{k}} \|_{1}, \\
& \text { since } \sum_{g=1}^{G} w_{g k}^{(r)}=1 \text { and } \mathbf{S}_{\mathbf{k}}=\frac{1}{n_{k}} \sum_{t=1}^{n_{k}} \mathbf{y}_{\mathbf{k t}} \mathbf{y}_{\mathbf{k t}}^{\mathbf{T}} . \text { Therefore, } \\
& Q_{\Omega_{k}}\left(\Theta ; \Theta^{(r)}\right)=\operatorname{tr}\left(\left(n_{k} \mathbf{S}_{\mathbf{k}}+\lambda_{2} \sum_{g=1}^{G} w_{g k}^{(r)} \boldsymbol{\Omega}_{\mathbf{0 g}}{ }^{(r)^{-1}}\right) \boldsymbol{\Omega}_{\mathbf{k}}\right)-\log \left|\boldsymbol{\Omega}_{\mathbf{k}}\right|\left(n_{k}+\lambda_{2}-p-1\right)+\lambda_{1}|| \boldsymbol{\Omega}_{\mathbf{k}} \|_{1} \propto \\
& \operatorname{tr}\left(\frac{n_{k} \mathbf{S}_{\mathbf{k}}+\lambda_{2} \sum_{g=1}^{G} w_{g k}^{(r)} \boldsymbol{\Omega}_{\mathbf{0 g}}^{(r)^{-1}}}{n_{k}+\lambda_{2}-p-1} \boldsymbol{\Omega}_{\mathbf{k}}\right)-\log \left|\boldsymbol{\Omega}_{\mathbf{k}}\right|+\frac{\lambda_{1}}{n_{k}+\lambda_{2}-p-1}\left\|\boldsymbol{\Omega}_{\mathbf{k}}\right\|_{1}
\end{aligned}
$$

which is solved using the glasso algorithm.

\section{Updates for $\mathrm{w}_{\mathrm{gk}}$ 's}

$$
w_{g k}^{(r+1)}=\frac{\pi_{g}^{(r+1)} p_{g}\left(\boldsymbol{\Omega}_{\mathbf{k}}^{(r+1)} ; \lambda_{2}, \boldsymbol{\Omega}_{\mathbf{0 g}}^{(r+1)}\right)}{\sum_{c=1}^{G} \pi_{c}^{(r+1)} p_{c}\left(\boldsymbol{\Omega}_{\mathbf{k}}^{(r+1)} ; \lambda_{2}, \boldsymbol{\Omega}_{\mathbf{0 c}}^{(r+1)}\right)}=\frac{\pi_{g}^{(r+1)} \exp \left(-\frac{\lambda_{2}}{2} \operatorname{tr}\left(\boldsymbol{\Omega}_{\mathbf{0 g}}^{(r+1)^{-1}} \boldsymbol{\Omega}_{\mathbf{k}}^{(r+1)}\right)\right)\left|\boldsymbol{\Omega}_{\mathbf{0 g}}{ }^{(r+1)}\right|^{-\frac{\lambda_{2}}{2}}}{\sum_{c=1}^{G} \pi_{c}^{(r+1)} \exp \left(-\frac{\lambda_{2}}{2} \operatorname{tr}\left(\boldsymbol{\Omega}_{\mathbf{0 c}}^{(r+1)^{-1}} \boldsymbol{\Omega}_{\mathbf{k}}^{(r+1)}\right)\right)\left|\boldsymbol{\Omega}_{\mathbf{0 c}}{ }^{(r+1)}\right|^{-\frac{\lambda_{2}}{2}}} .
$$




\section{Appendix B}

\section{Selecting the Number of Clusters}

For selecting the number of clusters, we used a gap statistic as proposed by Tibshirani et al. [2001]. Generally, the gap statistic compares the observed change in within-cluster dispersion of a clustering of subjects when specifying different numbers of clusters to what is expected under a corresponding null setting based on the observed data. For the setting in which we have data for $K$ subjects each with $n_{k}$ observations of $p$ variables, we calculate a gap statistic for the RCCM as follows:

1. Obtain the estimated clustering of subjects using RCCM when specifying $G$ total clusters for $G=2, \ldots, G_{\max }$ where $G_{\max }$ is the maximum number of clusters to be considered.

2. Calculate the GLasso estimate for the $k^{t h}$ subject using a small amount of penalization for $k=1,2, \ldots, K$. We used a tuning parameter value of $10^{-16}$ so that the optimal number of clusters was invariant to the choice of tuning parameters.

3. Calculate the $\log$ of the within-cluster variability for the observed data when specifying $G$ total clusters for $G=2, \ldots, G_{\max }$ as

$$
V_{G}=\log \left(\sum_{g=1}^{G} \sum_{k=1}^{K} \sum_{i=1}^{p} \sum_{j=1}^{p}\left(\omega_{k ; i, j}-\bar{\omega}_{g ; i, j}\right)^{2} \cdot z_{g k} /\left(G \cdot p^{2}\right)\right),
$$

where $\log (\cdot)$ is the natural $\log , z_{g k}=\mathbb{1}\{$ subject $k$ is in cluster $g\}$ is an indicator of the $k^{\text {th }}$ subject belonging to the $g^{\text {th }}$ cluster, $\bar{\omega}_{g ; i, j}=\sum_{k=1}^{K}\left(\omega_{k ; i, j} \cdot z_{g k}\right) / N_{g}$ is the average value of the GLasso estimate entry in the $i^{\text {th }}$ row and $j^{\text {th }}$ column for subjects in the $g^{\text {th }}$ cluster, and $N_{g}=\sum_{k=1}^{K} z_{g k}$ is the number of subjects in the $g^{\text {th }}$ cluster. We note that $V_{G}$ represents the $\log$ of the average variance of the precision matrix entries across subjects within each cluster.

4. Generate $B$ reference data sets for $K$ subjects and obtain the estimated clustering of subjects using RCCM when specifying $G$ total clusters for $G=2, \ldots, G_{\max }$. Specifically, generate $n_{k}$ observations for the $k^{t h}$ subject from a $\mathcal{N}_{p}\left(\mathbf{0}, \Omega_{b k}^{-1}\right)$. The entry in the $i^{\text {th }}$ row and $j^{\text {th }}$ column of $\Omega_{b k}^{-1}$ is generated from a uniform $\left(\min \left\{\omega_{k ; i, j}\right\}_{k=1}^{K}, \max \left\{\omega_{k ; i, j}\right\}_{k=1}^{K}\right)$ distribution where $\left\{\omega_{k ; i, j}\right\}_{k=1}^{K}$ are the GLasso entries for the observed data. We adjust the $\Omega_{b k}^{-1}$ matrix to make it positive definite if needed.

5. Implement the RCCM for each generated data set varying the specified number of clusters, and calculate $V_{G ; b}$ for $b=1,2, \ldots, B$ and $G=2, \ldots, G_{\max }$ as described in Equation (3).

6. Calculate the estimated gap statistics as

$$
\operatorname{Gap}(G)=\frac{1}{B} \sum_{b=1}^{B}\left(V_{G ; b}-V_{G}\right)=\bar{V}-V_{G} .
$$

7. Choose the optimal number of clusters as

$$
G^{*}=\arg \min \left\{G: \operatorname{Gap}(G) \geq \operatorname{Gap}(G+1)-\sigma_{G+1}\right\},
$$


A PREPRINT - OCTOBER 14, 2020

where $\sigma_{G+1}=\sqrt{\frac{\sum_{b=1}^{B}\left(V_{G ; b}-\bar{V}\right)^{2}}{B}} \cdot \sqrt{1+1 / B}$. 


\section{Appendix C}

Table 5: Accuracy of gap statistic across 100 simulations for selecting the correct number of clusters, where $G$ is the true number of clusters, Magnitude indicates whether the true precision matrices had entries high or low in magnitude, and Overlap is the proportion of overlapping edges across clusters.

\begin{tabular}{llrr}
\hline$G$ & Magnitude & Overlap & Accuracy \\
\hline 2 & High & 0.20 & 1.00 \\
& & 0.50 & 1.00 \\
& & 0.80 & 1.00 \\
\cline { 2 - 4 } & & 0.20 & 1.00 \\
& & 0.50 & 0.99 \\
& & 0.80 & 0.97 \\
\hline 3 & High & 0.20 & 1.00 \\
& & 0.50 & 1.00 \\
& & 0.80 & 1.00 \\
\cline { 2 - 3 } & Low & 0.20 & 1.00 \\
& & 0.50 & 1.00 \\
& & 0.80 & 0.99 \\
\hline
\end{tabular}


Table 6: Clustering performance of RCCM, Ward, and K-means clustering using 5-fold CV for tuning parameter selection. Results are for $G=2$ and 3 unbalanced groups, true precision matrices with entries large or small in magnitude, and group overlap being $0.20,0.50$, or 0.80 for each of 104 subjects averaged across 100 simulations. Ward clustering was based on a difference matrix of the Frobenius-norm differences between matrix estimates, while K-means clustering was based on vectorized GLasso matrix estimates.

\begin{tabular}{|c|c|c|c|c|c|}
\hline $\bar{G}$ & Magnitude & Overlap & Method & RI & $\mathrm{RI}_{\mathrm{adj}}$ \\
\hline \multirow[t]{18}{*}{2} & \multirow{9}{*}{ High } & \multirow{3}{*}{0.2} & RCCM & $1.000(0.000)$ & $1.000(0.000)$ \\
\hline & & & Ward \& GGL & $1.000(0.000)$ & $1.000(0.000)$ \\
\hline & & & GLasso \& K-means & $1.000(0.000)$ & $1.000(0.000)$ \\
\hline & & \multirow[t]{3}{*}{0.5} & $\mathrm{RCCM}$ & $1.000(0.000)$ & $1.000(0.000)$ \\
\hline & & & Ward \& GGL & $1.000(0.000)$ & $1.000(0.000)$ \\
\hline & & & GLasso \& K-means & $1.000(0.000)$ & $1.000(0.000)$ \\
\hline & & \multirow[t]{3}{*}{0.8} & $\mathrm{RCCM}$ & $1.000(0.000)$ & $1.000(0.000)$ \\
\hline & & & Ward \& GGL & $1.000(0.000)$ & $1.000(0.000)$ \\
\hline & & & GLasso \& K-means & $1.000(0.000)$ & $1.000(0.000)$ \\
\hline & \multirow[t]{9}{*}{ Low } & \multirow[t]{3}{*}{0.2} & $\mathrm{RCCM}$ & $1.000(0.000)$ & $1.000(0.000)$ \\
\hline & & & Ward \& GGL & $0.998(0.007)$ & $0.996(0.014)$ \\
\hline & & & GLasso \& K-means & $0.998(0.009)$ & $0.996(0.018)$ \\
\hline & & \multirow[t]{3}{*}{0.5} & $\mathrm{RCCM}$ & $1.000(0.003)$ & $0.999(0.005)$ \\
\hline & & & Ward \& GGL & $0.996(0.012)$ & $0.992(0.025)$ \\
\hline & & & GLasso \& K-means & $0.988(0.034)$ & $0.976(0.067)$ \\
\hline & & \multirow[t]{3}{*}{0.8} & RCCM & $0.992(0.015)$ & $0.983(0.031)$ \\
\hline & & & Ward \& GGL & $0.950(0.047)$ & $0.900(0.094)$ \\
\hline & & & GLasso \& K-means & $0.909(0.152)$ & $0.810(0.324)$ \\
\hline \multirow[t]{18}{*}{3} & \multirow[t]{9}{*}{ High } & \multirow[t]{3}{*}{0.2} & RCCM & $1.000(0.000)$ & $1.000(0.000)$ \\
\hline & & & Ward \& GGL & $1.000(0.000)$ & $1.000(0.000)$ \\
\hline & & & GLasso \& K-means & $0.853(0.124)$ & $0.691(0.260)$ \\
\hline & & \multirow[t]{3}{*}{0.5} & $\mathrm{RCCM}$ & $1.000(0.000)$ & $1.000(0.000)$ \\
\hline & & & Ward \& GGL & $1.000(0.000)$ & $1.000(0.000)$ \\
\hline & & & GLasso \& K-means & $0.872(0.124)$ & $0.733(0.259)$ \\
\hline & & \multirow[t]{3}{*}{0.8} & $\mathrm{RCCM}$ & $1.000(0.000)$ & $1.000(0.000)$ \\
\hline & & & Ward \& GGL & $0.999(0.003)$ & $0.998(0.007)$ \\
\hline & & & GLasso \& K-means & $0.965(0.088)$ & $0.928(0.180)$ \\
\hline & \multirow[t]{9}{*}{ Low } & \multirow[t]{3}{*}{0.2} & $\mathrm{RCCM}$ & $0.999(0.003)$ & $0.998(0.007)$ \\
\hline & & & Ward \& GGL & $0.995(0.009)$ & $0.989(0.020)$ \\
\hline & & & GLasso \& K-means & $0.934(0.100)$ & $0.863(0.206)$ \\
\hline & & \multirow[t]{3}{*}{0.5} & RCCM & $0.999(0.003)$ & $0.998(0.007)$ \\
\hline & & & Ward \& GGL & $0.991(0.012)$ & $0.980(0.026)$ \\
\hline & & & GLasso \& K-means & $0.929(0.094)$ & $0.855(0.190)$ \\
\hline & & \multirow[t]{3}{*}{0.8} & RCCM & $0.989(0.019)$ & $0.975(0.041)$ \\
\hline & & & Ward \& GGL & $0.945(0.040)$ & $0.880(0.086)$ \\
\hline & & & GLasso \& K-means & $0.896(0.098)$ & $0.788(0.192)$ \\
\hline
\end{tabular}


Table 7: Comparison of method performances for $G=2$ unbalanced groups containing 67 and 37 subjects. Results are for observations of $p=10$ variables and $n=177$ observations for each subject using 5-fold CV for tuning parameter selection, averaged across 100 simulations. Grouplevel performance measures for GLasso are missing since the method does not yield cluster-level estimates.

\begin{tabular}{|c|c|c|c|c|c|c|c|c|}
\hline Magnitude & Overlap & Method & $\mathrm{TPR}_{\mathrm{g}}$ & $\mathrm{FPR}_{\mathrm{g}}$ & $\mathrm{PPV}_{\mathrm{g}}$ & $\mathrm{TPR}_{\mathrm{k}}$ & $\mathrm{FPR}_{\mathrm{k}}$ & $\mathrm{PPV}_{\mathrm{k}}$ \\
\hline \multirow[t]{18}{*}{ High } & \multirow[t]{6}{*}{0.2} & $\mathrm{RCCM}$ & 0.999 & 0.210 & 0.507 & 1.000 & 0.626 & 0.252 \\
\hline & & & $(0.007)$ & $(0.041)$ & $(0.046)$ & $(0.002)$ & $(0.029)$ & $(0.014)$ \\
\hline & & Ward \& GGL & 1.000 & 0.322 & 0.400 & 1.000 & 0.451 & 0.319 \\
\hline & & & $(0.000)$ & $(0.049)$ & $(0.037)$ & $(0.005)$ & $(0.010)$ & $(0.011)$ \\
\hline & & GLasso \& K-means & & & & 1.000 & 0.452 & 0.318 \\
\hline & & & & & & $(0.005)$ & $(0.010)$ & $(0.011)$ \\
\hline & \multirow[t]{6}{*}{0.5} & $\overline{\mathrm{RCCM}}$ & 1.000 & 0.250 & 0.501 & 1.000 & 0.626 & 0.286 \\
\hline & & & $(0.000)$ & $(0.018)$ & $(0.019)$ & $(0.000)$ & $(0.056)$ & $(0.016)$ \\
\hline & & Ward \& GGL & 1.000 & 0.247 & 0.507 & 1.000 & 0.437 & 0.364 \\
\hline & & & $(0.000)$ & $(0.044)$ & $(0.044)$ & $(0.000)$ & $(0.008)$ & $(0.004)$ \\
\hline & & GLasso \& K-means & & & & 1.000 & 0.438 & 0.363 \\
\hline & & & & & & $(0.000)$ & $(0.008)$ & $(0.004)$ \\
\hline & \multirow[t]{6}{*}{0.8} & $\mathrm{RCCM}$ & 0.947 & 0.247 & 0.508 & 1.000 & 0.736 & 0.255 \\
\hline & & & $(0.000)$ & $(0.021)$ & $(0.021)$ & $(0.000)$ & $(0.071)$ & $(0.020)$ \\
\hline & & Ward \& GGL & 0.951 & 0.304 & 0.459 & 1.000 & 0.444 & 0.360 \\
\hline & & & $(0.013)$ & $(0.043)$ & $(0.037)$ & $(0.000)$ & $(0.007)$ & $(0.004)$ \\
\hline & & GLasso \& K-means & & & & 1.000 & 0.446 & 0.359 \\
\hline & & & & & & $(0.000)$ & $(0.007)$ & $(0.004)$ \\
\hline \multirow[t]{18}{*}{ Low } & \multirow[t]{6}{*}{0.2} & RCCM & 0.996 & 0.438 & 0.370 & 0.999 & 0.559 & 0.287 \\
\hline & & & $(0.015)$ & $(0.236)$ & $(0.168)$ & $(0.006)$ & $(0.188)$ & $(0.083)$ \\
\hline & & Ward \& GGL & 0.990 & 0.387 & 0.369 & 0.989 & 0.429 & 0.350 \\
\hline & & & $(0.023)$ & $(0.141)$ & $(0.130)$ & $(0.015)$ & $(0.151)$ & $(0.128)$ \\
\hline & & GLasso \& K-means & & & & 0.803 & 0.212 & 0.443 \\
\hline & & & & & & $(0.046)$ & $(0.048)$ & $(0.042)$ \\
\hline & \multirow[t]{6}{*}{0.5} & $\mathrm{RCCM}$ & 0.989 & 0.476 & 0.390 & 0.992 & 0.586 & 0.314 \\
\hline & & & $(0.023)$ & $(0.245)$ & $(0.157)$ & $(0.013)$ & $(0.186)$ & $(0.081)$ \\
\hline & & Ward \& GGL & 0.849 & 0.341 & 0.427 & 0.877 & 0.376 & 0.412 \\
\hline & & & $(0.066)$ & $(0.160)$ & $(0.151)$ & $(0.046)$ & $(0.174)$ & $(0.151)$ \\
\hline & & GLasso \& K-means & & & & 0.682 & 0.200 & 0.464 \\
\hline & & & & & & $(0.047)$ & $(0.041)$ & $(0.034)$ \\
\hline & \multirow[t]{6}{*}{0.8} & RCCM & 0.990 & 0.789 & 0.307 & 0.993 & 0.810 & 0.314 \\
\hline & & & $(0.032)$ & $(0.139)$ & $(0.043)$ & $(0.008)$ & $(0.105)$ & $(0.029)$ \\
\hline & & Ward \& GGL & 0.868 & 0.482 & 0.507 & 0.789 & 0.486 & 0.390 \\
\hline & & & $(0.150)$ & $(0.338)$ & $(0.275)$ & $(0.093)$ & $(0.168)$ & $(0.062)$ \\
\hline & & GLasso \& K-means & & & & 0.634 & 0.226 & 0.514 \\
\hline & & & & & & $(0.050)$ & $(0.049)$ & $(0.037)$ \\
\hline
\end{tabular}


Table 8: Comparison of method performances for $G=3$ unbalanced groups containing 61, 24, and 19 subjects. Results are for observations of $p=10$ variables and $n=177$ observations for each subject using 5-fold CV for tuning parameter selection, averaged across 100 simulations. Grouplevel performance measures for GLasso are missing since the method does not yield cluster-level estimates.

\begin{tabular}{|c|c|c|c|c|c|c|c|c|}
\hline Magnitude & Overlap & Method & $\mathrm{TPR}_{\mathrm{g}}$ & $\mathrm{FPR}_{\mathrm{g}}$ & $\mathrm{PPV}_{\mathrm{g}}$ & $\mathrm{TPR}_{\mathrm{k}}$ & $\mathrm{FPR}_{\mathrm{k}}$ & $\mathrm{PPV}_{\mathrm{k}}$ \\
\hline \multirow[t]{18}{*}{ High } & \multirow[t]{6}{*}{0.2} & \multirow[t]{2}{*}{$\overline{\mathrm{RCCM}}$} & 0.913 & 0.102 & 0.647 & 1.000 & 0.620 & 0.229 \\
\hline & & & $(0.000)$ & $(0.005)$ & $(0.010)$ & $(0.000)$ & $(0.007)$ & $(0.002)$ \\
\hline & & \multirow[t]{2}{*}{ Ward \& GGL } & 0.940 & 0.298 & 0.395 & 1.000 & 0.448 & 0.292 \\
\hline & & & $(0.025)$ & $(0.030)$ & $(0.025)$ & $(0.000)$ & $(0.007)$ & $(0.003)$ \\
\hline & & \multirow[t]{2}{*}{ GLasso \& K-means } & & & & 1.000 & 0.449 & 0.291 \\
\hline & & & & & & $(0.000)$ & $(0.007)$ & $(0.003)$ \\
\hline & \multirow[t]{6}{*}{0.5} & \multirow[t]{2}{*}{$\mathrm{RCCM}$} & 1.000 & 0.092 & 0.655 & 0.973 & 0.619 & 0.224 \\
\hline & & & $(0.000)$ & $(0.006)$ & $(0.016)$ & $(0.005)$ & $(0.007)$ & $(0.002)$ \\
\hline & & \multirow[t]{2}{*}{ Ward \& GGL } & 1.000 & 0.277 & 0.388 & 0.960 & 0.444 & 0.285 \\
\hline & & & $(0.000)$ & $(0.033)$ & $(0.028)$ & $(0.005)$ & $(0.007)$ & $(0.003)$ \\
\hline & & \multirow{2}{*}{\multicolumn{2}{|c|}{ GLasso \& K-means }} & & & 0.960 & 0.445 & 0.284 \\
\hline & & & & & & $(0.005)$ & $(0.007)$ & $(0.003)$ \\
\hline & \multirow[t]{6}{*}{0.8} & \multirow[t]{2}{*}{ RCCM } & 0.963 & 0.102 & 0.723 & 0.999 & 0.772 & 0.274 \\
\hline & & & $(0.045)$ & $(0.025)$ & $(0.060)$ & $(0.005)$ & $(0.040)$ & $(0.019)$ \\
\hline & & \multirow[t]{2}{*}{ Ward \& GGL } & 0.998 & 0.366 & 0.432 & 0.992 & 0.463 & 0.384 \\
\hline & & & $(0.012)$ & $(0.056)$ & $(0.034)$ & $(0.026)$ & $(0.019)$ & $(0.028)$ \\
\hline & & \multirow[t]{2}{*}{ GLasso \& K-means } & & & & 0.997 & 0.463 & 0.385 \\
\hline & & & & & & $(0.009)$ & $(0.017)$ & $(0.027)$ \\
\hline \multirow[t]{18}{*}{ Low } & \multirow[t]{6}{*}{0.2} & \multirow[t]{2}{*}{$\mathrm{RCCM}$} & 0.973 & 0.455 & 0.340 & 0.994 & 0.551 & 0.290 \\
\hline & & & $(0.076)$ & $(0.204)$ & $(0.128)$ & $(0.012)$ & $(0.170)$ & $(0.075)$ \\
\hline & & \multirow[t]{2}{*}{ Ward \& GGL } & 0.957 & 0.380 & 0.361 & 0.969 & 0.393 & 0.365 \\
\hline & & & $(0.103)$ & $(0.124)$ & $(0.109)$ & $(0.026)$ & $(0.128)$ & $(0.116)$ \\
\hline & & \multirow{2}{*}{\multicolumn{2}{|c|}{ GLasso \& K-means }} & & & 0.696 & 0.182 & 0.446 \\
\hline & & & & & & $(0.031)$ & $(0.020)$ & $(0.019)$ \\
\hline & \multirow[t]{6}{*}{0.5} & \multirow[t]{2}{*}{$\mathrm{RCCM}$} & 0.983 & 0.724 & 0.280 & 0.993 & 0.753 & 0.275 \\
\hline & & & $(0.035)$ & $(0.140)$ & $(0.044)$ & $(0.010)$ & $(0.111)$ & $(0.029)$ \\
\hline & & \multirow[t]{2}{*}{ Ward \& GGL } & 0.974 & 0.712 & 0.280 & 0.947 & 0.634 & 0.302 \\
\hline & & & $(0.047)$ & $(0.107)$ & $(0.041)$ & $(0.035)$ & $(0.121)$ & $(0.033)$ \\
\hline & & \multirow[t]{2}{*}{ GLasso \& K-means } & & & & 0.763 & 0.249 & 0.470 \\
\hline & & & & & & $(0.041)$ & $(0.045)$ & $(0.040)$ \\
\hline & \multirow[t]{6}{*}{0.8} & \multirow[t]{2}{*}{ RCCM } & 0.964 & 0.762 & 0.295 & 0.985 & 0.774 & 0.312 \\
\hline & & & $(0.046)$ & $(0.126)$ & $(0.036)$ & $(0.013)$ & $(0.108)$ & $(0.029)$ \\
\hline & & \multirow[t]{2}{*}{ Ward \& GGL } & 0.834 & 0.498 & 0.413 & 0.789 & 0.472 & 0.378 \\
\hline & & & $(0.145)$ & $(0.252)$ & $(0.191)$ & $(0.079)$ & $(0.124)$ & $(0.045)$ \\
\hline & & \multirow[t]{2}{*}{ GLasso \& K-means } & & & & 0.602 & 0.207 & 0.509 \\
\hline & & & & & & $(0.050)$ & $(0.045)$ & $(0.034)$ \\
\hline
\end{tabular}

